\title{
A signal detection model predicts the effects of set size on visual search accuracy for feature, conjunction, triple conjunction, and disjunction displays
}

\author{
MIGUEL P. ECKSTEIN \\ Cedars Sinai Medical Center, Los Angeles, California \\ JAMES P. THOMAS \\ University of Califormia, Los Angeles, Califormia \\ JOHN PALMER \\ University of Washington, Seattle, Washington \\ and \\ STEVEN S. SHIMOZAKI \\ University of Rochester, Rochester, New York
}

\begin{abstract}
Recently, quantitative models based on signal detection theory have been successfully applied to the prediction of human accuracy in visual search for a target that differs from distractors along a single attribute (feature search). The present paper extends these models for visual search accuracy to multidimensional search displays in which the target differs from the distractors along more than one feature dimension (conjunction, disjunction, and triple conjunction displays). The model assumes that each element in the display elicits a noisy representation for each of the relevant feature dimensions. The observer combines the representations across feature dimensions to obtain a single decision variable, and the stimulus with the maximum value determines the response. The model accurately predicts human experimental data on visual search accuracy in conjunctions and disjunctions of contrast and orientation. The model accounts for performance degradation without resorting to a limited-capacity spatially localized and temporally serial mechanism by which to bind information across feature dimensions.
\end{abstract}

Visual search for a target among a set of distractors has been extensively studied by a large number of investigators. Typically, the observer's reaction time for finding the target is measured as a function of the number of distractors (set size) in the display. When the target and the distractors differ along one physical dimension or stimulus attribute (e.g., length, orientation, color, brightness, etc.), the search task is known as a feature search. A com-

Some of the results of this paper were presented at the Association for Research in Visual Ophthalmology 1995 and 1996 Annual Meeting in Ft. Lauterdale, Florida (Eckstein et al., 1995, 1996). This research was supported by NIH-RO1 HL53455 and NASA NCC 2-1027. We thank Dawn Aiken for the data collected at the University of Washington. M.P.E. thanks J. Cortazar and D. J. Shadow for help with the completion of the work, Dante Spinetta and Emmanuel Horvilleur for help with the Monte Carlo ensemble simulations, and Dick Swensson and Neil Macmillan for very helpful suggestions (including "the feature combination rule") to improve the quality and presentation of the paper. Correspondence concerning this article should be addressed to $\mathbf{M}$. P. Eckstein, Department of Medical Physics and Imaging, Cedars Sinai Medical Center, 8700 Beverly Blvd., Davis 6065, Los Angeles, CA 90048-1865 (e-mail: miguel@medphysics.csmc.edu or miguel@) vision.arc.nasa.gov). On-line fitting of signal detection theory based models to feature and multidimensional displays are available at http://www.csmc.edu/medphys/Cyberfits/html. mon finding for feature search is that reaction times are independent of the number of distractors (small set-size effect) in the display, which is commonly interpreted as evidence for unlimited capacity parallel processing of all the items in the display. On the other hand, when the target/ distractors discriminability is defined along two feature dimensions, so that the target differs from two types of distractors by a different feature dimension (e.g., a tilted long line among tilted short lines and vertical long lines), the search task is known as a conjunction search. In this case, a common finding is that reaction times increase as the number of distractors in the display increases (large setsize effect), which is taken as evidence of serial processing (e.g., Treisman \& Gelade, 1980; Treisman \& Gormican, 1988).

The dichotomy in results between feature (parallel processing) and conjunction (serial processing) search was interpreted by Treisman (feature integration theory; Treisman \& Gelade, 1980) as evidence that integration or binding of information across features can only be accomplished through a spatially localized mechanism (visual attention) that operates in serial fashion. In feature integration theory, the visual field is first analyzed by a series of spatiotopically organized maps, called feature maps, 
encoding the presence of a stimulus attribute or feature (e.g., color, motion, orientation, etc.). Each feature map independently signals the presence of a specific feature (redness, tilted, long, etc.) in parallel across the visual field. Therefore, if the visual search task is defined by a feature (presence of tiltedness), the time to find the target is independent of the number of elements in the display.

On the other hand, in order to combine activity or information across different feature maps, visual attention must focus on a localized region of the feature maps. Visual attention is inherently spatially localized and temporally serial.

Further experiments (Duncan \& Humphreys, 1989) showed that when the similarity of the target and the distractors is high, the set-size effect is large even with feature displays. Treisman explained such findings by stating that when the target/distractor similarity is high, visual attention is needed to perform fine discrimination (Treisman \& Gormican, 1988).

Recently, other studies have shown that other lower level factors affect visual search performance. Increasing element retinal eccentricity has been shown to increase set-size effects (Carrasco, Evert, Chang, \& Katz, 1995; Geisler \& Chou, 1995). Increasing element density has been shown to increase set-size effects, because of an increase in lateral masking (Carrasco et al., 1995). Experiments have also shown that the number of eye movements increases with increasing target/distractor similarity (Zelinsky, Sheinberg, \& Bulthoff, 1993). These results suggest that, under free viewing (eye movements allowed), it becomes hard to separate set-size effects that are due to the increasing number of eye movements from those that are due to the serial allocation of visual attention. Many of the search time studies have not carefully controlled for all these low-level factors that may be confounded with any possible set-size effects that are due to the capacity limitations of attentional nature. An additional limitation of response time studies is that it is difficult to keep observers' accuracy levels constant, so as not to have differential speed/accuracy tradeoffs across set-size conditions. The inability to keep performance at a constant level across set-size conditions might obscure any interpretations about capacity limitations.

In order to control for some of these lower level factors and for differential speed/accuracy tradeoffs, many authors have chosen to use a visual search accuracy study (Bergen \& Julesz, 1983a, 1983b; Palmer, 1994a; Palmer, Ames, \& Lindsey, 1993). In this type of study, the display is briefly presented. The brief presentation precludes eye movements by the observer during the trials. The ability to correctly determine whether the target is present (yes/no design) or which of $M$ displays contains the target (alternative forced-choice [AFC] design) is measured. The investigator manipulates the number of distractors in the display for the different experimental conditions. The rationale for the accuracy study is that performance degradation with increasing set size may be used to draw conclusions about capacity limitations. The serial model makes explicit predictions for set-size effects in visual search accuracy studies. Bergen and Julesz (1983a, 1983b) used this method to study search for an L among Ts. Their results were consistent with a temporally serial mechanism.

More recently, investigators have taken into consideration the inherent noise in visual processing. The presence of noise in the encoding of visual properties is supported by physiological studies measuring the statistical reliability of the responses of cells (Tolhurst, Movshson, \& Dean, 1982). As will be discussed in detail, the inclusion of noise in the encoding of each element will produce setsize effects in visual search accuracy.

Palmer et al. (1993; Palmer, 1994a) have shown that performance degradation as a function of the number of distractors in feature displays can be predicted by a simple model (decision integration hypothesis) that assumes that the observer has noisy representations of the target and the distractors (Palmer, 1994a; Palmer et al., 1993; Shaw, 1980) and uses the maximum response as the decision variable. This model is based on the widely used signal detection theory (SDT; Green \& Swets, 1966; Swets, 1964) first applied by Tanner and Swets (1954) and Tanner (1961). The model does not assume any change in the quality of the representation of each individual element as a function of target/distractor similarity or increasing number of distractors. The model has been successfully applied in predicting the effect of number of distractors on performance in search tasks in which the target was defined by a variety of properties-disk luminance, blob luminance, blob color, disk size, ellipse orientation (Palmer, 1994a), letter type (Bennett \& Jaye, 1995), target speed (Verghese \& Stone, 1995) — and in tasks involving the detection of contrast-defined targets on a variety of backgrounds (Burgess \& Ghandeharian, 1984; Eckstein \& Whiting, 1996; Swensson \& Judy, 1981).

The purpose of this study is to extend the principles of SDT to visual search accuracy in multidimensional search displays and to report quantitative predictions for performance degradation as a function of target/distractor similarity for three commonly used multidimensional search displays: conjunction, triple conjunction, and disjunction (two-dimensional [2-D] feature) displays. The model is used to fit data on human visual search accuracy for conjunction and disjunction (2-D feature) displays collected by Aiken and Palmer (1992). The methodology and quantitative predictions that are presented can be used to rigorously test whether the results of visual search accuracy studies can be accounted for by the inherent noise in the visual system or whether capacity limitations (e.g., serial processing) need to be invoked. Before presenting of the theory for multidimensional displays, we discuss the theory for single-dimension (feature) displays.

\section{ONE-DIMENSIONAL (FEATURE) DISPLAYS}

\section{Stimuli and Experimental Tasks}

One important aspect of the work presented in this study is that the predictions of the model are independent of the dimension manipulated in the experiment. 


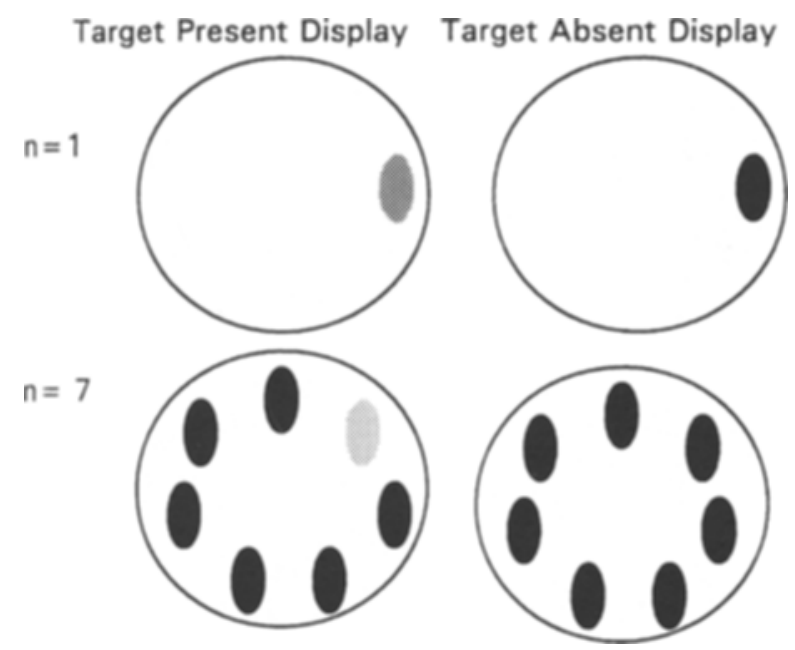

Figure 1. Feature search task for a two-alternative forcedchoice defined with respect to contrast. One alternative contains the target (brighter ellipse) and $n-1$ distractors (dimmer ellipse); the other alternative contains $n$ distractors. The observers' task is to correctly identify the alternative with the target. Examples for $n=1$ and $n=7$.

The dimensions could be luminance, orientation, length, hue, motion, speed, and so forth. The important parameter that will quantitatively determine the effect of the number of distractors is performance $\left(P_{\mathrm{c}}\right.$, proportion correct) in identifying a single target from a single distractor along the relevant dimension. As will be discussed later, an underlying internal target/distractor discriminability is associated with the $P_{\mathrm{c}}$. The observer's internal target/distractor discriminability can be changed by using different experimental manipulations. For example, one can change the physical appearance of the distractor to make it more discriminable from the target. On the other hand, one could shorten the time of presentation of the display, which might reduce performance in discriminating a single target from the distractors, effectively reducing the internal target/distractor discriminability. The essential point is that the feature attribute that distinguishes the distractors from the target and the method of manipulating the discriminability are irrelevant to the model's predictions of performance degradation as a function of set size. The derived results apply to any attributes and to any methods of manipulating discriminability. The only restriction is that, as the number of distractors is increased, any factors affecting discrimination of a single item (such as presentation time) must be kept constant.

In this paper, we consider results for an AFC accuracy design; however, the results can be generalized to yes/no (Palmer et al., 1993) and rating type designs. In an M-AFC design, $M$ displays or alternatives are presented to the observer: one alternative containing the target and $M-1$ alternatives with nontargets. The task of the observer is to correctly identify the display or the alternative containing the target. In this paper, we consider studies in which the observer knows the target a priori. The alternatives are presented either simultaneously or sequentially through time. In the design used by Palmer and in the studies presented in this paper, the observer responds without a response deadline. However, in order to control the response time, one might include a response deadline.

The number of distractors is manipulated so that the target-present alternative contains the target and $n-1$ distractors and the target-absent alternative contains the $n$ distractors. Figure 1 illustrates the task of searching for a bright ellipse (target) among dimmer ellipses (distractors) for set sizes $n=1$ and $n=7$ and for two alternatives $(M=2)$.

\section{Theoretical Principles}

The basic assumption of SDT is that each element in the display elicits an internal response in the observer. The internal response to the same element will vary from trial to trial, owing to the internal noise. Possible sources of internal noise include fluctuations in the firing of the cells (Barlow, 1957; Tolhurst et al., 1982) and variability in the decision criterion (Wickelgren, 1968). On average, the target elicits a different internal response than do the distractors. For example, if the target is brighter than the distractors, the internal response to the target will be, on average, larger than that to the distractor. However, owing to the internal noise, the distractor might elicit a larger response than the target in some trials. On each trial, the observer is assumed to monitor $n$ internal responses to the $n$ distractors in the target-absent display and another $n$ internal responses corresponding to the $n-1$ distractors and the target in the target-present display. The observer then uses the maximum response among the $n$ internal responses for each display as the decision variable. If the maximum response for Display 1 is larger than the maximum response for Display 2, then choose Display 1; otherwise, choose Display 2 (Figure 2). Although the maximum response rule is not the ideal Bayesian strategy (Pelli, 1985), it has been shown to predict human results for a number of visual tasks (Palmer, 1994a; Palmer et al., 1993; Swensson \& Judy, 1981 ), and it sometimes approximates the optimal decision rule (Nolte \& Jaarsma, 1966).

If we define $t(x)$ as the probability of the target response taking a value $x, d(x)$ as the probability of the distractor taking a value $x, T(x)$ as the cumulative probability of the target taking a value less than $x, D(x)$ as the cumulative probability of the distractor taking a value less than $x, n$ as the number of distractors in the target-absent display (set size), and $M$ as the number of response alternatives or intervals, the probability of correct identification of the display containing the target (for derivation, see Appendix A) can be written as

$$
\begin{aligned}
P_{\mathrm{c}}(n, M)= & \int_{-\infty}^{+\infty}\left[t(x) D^{n-1}(x)+(n-1) d(x) D^{n-2}(x) T(x)\right] \\
& \cdot\left[D^{n}(x)\right]^{M-1} d x .
\end{aligned}
$$

With the assumptions that the internal noise of the observer is Gaussian distributed and that the target distrib- 


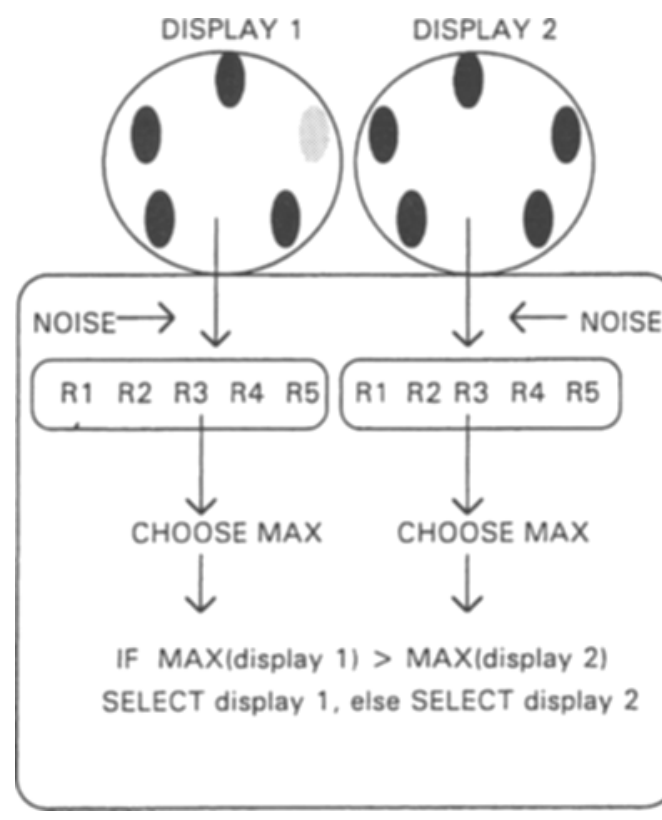

OBSERVER

Figure 2. Decision strategy for observer with maximum response rule (Palmer, Ames, \& Lindsey, 1993; Swensson \& Judy, 1981). Each element in the two alternatives elicits an internal response in the observer. The responses are subject to random fluctuations owing to internal neural noise. The observer chooses the maximum response per alternative to use as the decision variable and selects the alternative associated with the highest maximum response.

ution has the same variance as the distractor distribution but a higher mean $\left(u_{0}\right)$, the probability distibutions of the internal responses can be parameterized as a function of $d_{\mathrm{o}}^{\prime}$, defined as the distance between the center of the two distributions divided by the standard deviation $\left(\sigma_{0}\right)$. For the case of two response intervals or alternatives $(M=2)$ investigated in this paper, $P_{\mathrm{c}}$ is given by

$$
\begin{aligned}
P_{\mathrm{c}}(n, M= & \left.2, d_{\mathrm{o}}^{\prime}\right) \\
= & \int_{-\infty}^{+\infty}\left[g\left(x-d_{\mathrm{o}}^{\prime}\right) G^{n-1}(x)\right. \\
& \left.+(n-1) g(x) G^{n-2}(x) G\left(x-d_{\mathrm{o}}^{\prime}\right)\right] G^{n}(x) d x,
\end{aligned}
$$

where

$$
d_{\mathrm{o}}^{\prime}=\frac{u_{\mathrm{o}}}{\sigma_{\mathrm{o}}}
$$

$g(x)$ is the Gaussian probability that the element value takes a value $x$ and $G(x)$ is the cumulative Gaussian probability that the element distribution takes a value less than $x$.

The observers' internal discriminability between the target and the distractor is described by $d_{\mathrm{o}}^{\prime}$. In practice, $P_{\mathrm{c}}$ for a given number of alternatives and distractors is measured, and a corresponding $d_{\mathrm{o}}^{\prime}$ is inferred from Equation 2 .

\section{Representing Target/Distractor Similarity}

A large number of experiments have shown that increasing the similarity between the target and the dis- tractor will reduce search efficiency, producing steeper slopes in search time studies. These experiments have manipulated color (Farmer \& Taylor, 1980; Nagy \& Sanchez, 1990), curvature of lines (Treisman \& Gormican, 1988), letters (Bergen \& Julesz, 1983a, 1983b; Duncan \& Humphreys, 1989; Estes, 1972; Pashler, 1987; Pavel, Econopouly, \& Landy, 1992; Treisman \& Gelade, 1980; Verghese \& Nakayama, 1994; von Grünau, Dubé, \& Galera, 1994), and so forth. Palmer (1995) has performed search accuracy studies in which the target was a disk with a higher luminance value than the distractors. His results show larger set-size effects (performance degradation from one and seven distractors) for the conditions with smaller target/distractor differences in luminance.

In the context of SDT, changing the physical difference between the target and the distractors along the relevant feature dimensions will change the internal discriminability between the target and the distractor $\left(d_{0}^{\prime}\right)$. The exact relationship between the change in physical difference (e.g., percentage of orientation difference or contrast difference) and the internal discriminability between target and distractor will depend on the feature dimension manipulated. For example $d_{\mathrm{o}}^{\prime}$ might be a linear function, a power function, a $\log$ function, or some other function of the physical difference between the target and the distractor along the relevant feature dimension. However, given a certain level of target/distractor internal discriminability $\left(d_{\mathrm{o}}^{\prime}\right)$, the effect of the number of distractors 
on performance can be obtained by evaluating Equation 2 for the value of $d_{o}^{\prime}$.

Figure 3 (left panels) shows the probability distributions for the maximum response of all elements in the target-present display (target and $n-1$ distractors) and the maximum response of all elements in the target-absent display ( $n$ distractors) for 1,5 , and 30 distractors. The target/distractor discriminability, $d_{0}^{\prime}$, is 2.0 .

Overlap between these two distributions is indirectly related to the probability of correctly identifying the targetpresent display and might give the reader some intuition as to how performance is affected by increasing the set size.

Figure 3 (left panels) illustrates how, as the number of distractors increases, the overlap of the probability distributions increases, effectively reducing the $P_{\mathrm{c}}$ for discriminating the target-present from the target-absent display. Although both the target-absent and the target-present displays contain distractors, the net effect of adding distractors is to increase the probability that the maximum response in the target-absent display will exceed the maximum response in the target-present display. This behavior is a consequence of the internal noise and of the maximum response decision strategy and not of any reduction in the underlying internal discriminability $\left(d_{0}^{\prime}\right)$ between a single target and distractor.

Figure 3 (right panels) shows the probability distributions of the maximum response for a case in which the target/distractor discriminability is high $\left(d_{\mathrm{o}}^{\prime}=4.5\right)$. Increasing the set size for the high target/distractor discriminability results in a smaller increase in the distributions' overlap than was the case for the lower target/ distractor discriminability $\left(d_{0}^{\prime}=2.0\right)$. Therefore, the net effect of increasing the number of distractors interacts with the discriminability between the target and a single distractor.

Figure 4 shows $P_{\mathrm{c}}$ as a function of the number of distractors in the display for five different levels of internal target/distractor discriminability $\left(d_{\mathrm{o}}^{\prime}\right)$. For very high levels of discriminability $\left(d_{\mathrm{o}}^{\prime}=4.5\right), P_{\mathrm{c}}$ remains approximately constant as a function of number of distractors. When target/distractor discriminability $\left(d_{\mathrm{o}}^{\prime}\right)$ is decreased, the change in $P_{\mathrm{c}}$ with number of distractors increases. ${ }^{1}$ Table 1 lists the obtained values of $P_{\mathrm{c}}$ as a function of number of distractors $(n)$ for different levels of target/ distractor discriminability $\left(d_{0}^{\prime}\right)$, based on the numerical evaluation of Equation 2.

\section{MULTIDIMENSIONAL SEARCH DISPLAYS: BASIC THEORY}

The SDT model can be extended to displays in which the target differs from the distractors along more than one physical attribute or feature dimension (multidimensional; Eckstein, Thomas, Palmer, \& Shimozaki, 1996; Eckstein, Thomas, Shimozaki, \& Whiting, 1995; Pavel et al., 1992). In the context of SDT, we assume that each of the feature dimensions of interest elicits an in- dependent noisy internal value in the observer. In this way, each element in the display elicits $f$ internal responses corresponding to the $f$ feature dimensions. For the case of two feature dimensions, we can represent the distributions of internal responses associated with the target and distractors by plotting a probability surface in $x-y$ Cartesian coordinates (Figure 5). The $x$-axis corresponds to the probability distribution of the internal response to the display elements along the first dimension (e.g., orientation), and the $y$-axis corresponds to the probability distribution of the internal response to the display elements along the second dimension (e.g., contrast).

The specific distractor configuration corresponds to a typical conjunction display (Treisman \& Gelade, 1980) in which the target differs from each of the two distractors along one feature dimension. In this paper, we investigate models that combine the information across the two dimensions to create a new variable $Z=f\left(x_{1}, x_{2}\right)$. In this way, each element in the display will be associated with a value along the new decision variable $Z$. The decision process then remains the same: If the maximum response to all the elements in Display 1 is greater than the maximum response to all the elements in Display 2, then choose Display 1; otherwise, choose Display 2 . Figure 6 illustrates the decision rule for a multidimensional display that includes two relevant feature dimensions.

The specific mathematical form $f\left(x_{1}, x_{2}\right)$ of the function used by human observers to combine information across dimensions $x_{1}$ and $x_{2}$ is unknown. In this paper, we consider two possible decision rules: (1) the maximum of the linear combination of the responses along the feature dimensions (max-linear) and (2) the maximum response among the elements' minimum response between/among the different feature dimensions ( $\max -\mathrm{min}$ ).

In order to generate predictions of the $P_{\mathrm{c}}$ for the multidimensional displays, two steps are required: (1) to obtain mathematical expressions by which to calculate the effective target/distractor discriminability along the new decision variable after the combination of information across feature dimensions, and (2) to obtain an expression that takes into account the possibility of different types of distractors with different associated target/ distractor discriminabilities along the new decision variable. Appendices $B$ and $C$ develop in detail the mathematical foundations for the general framework; however, with the introduction of a number of assumptions, the predictions of the model are greatly simplified.

\section{Combining Information Across Feature Dimensions}

The first step required to develop predictions for multidimensional displays is to be able to calculate the effective target/distractor discriminability after combination of information across feature dimensions.

Max-linear decision rule. In the multidimensional displays, the target and the distractors elicit a number of noisy responses $\left(x_{1}, x_{3}, \ldots x_{f}\right)$. The max-linear decision 

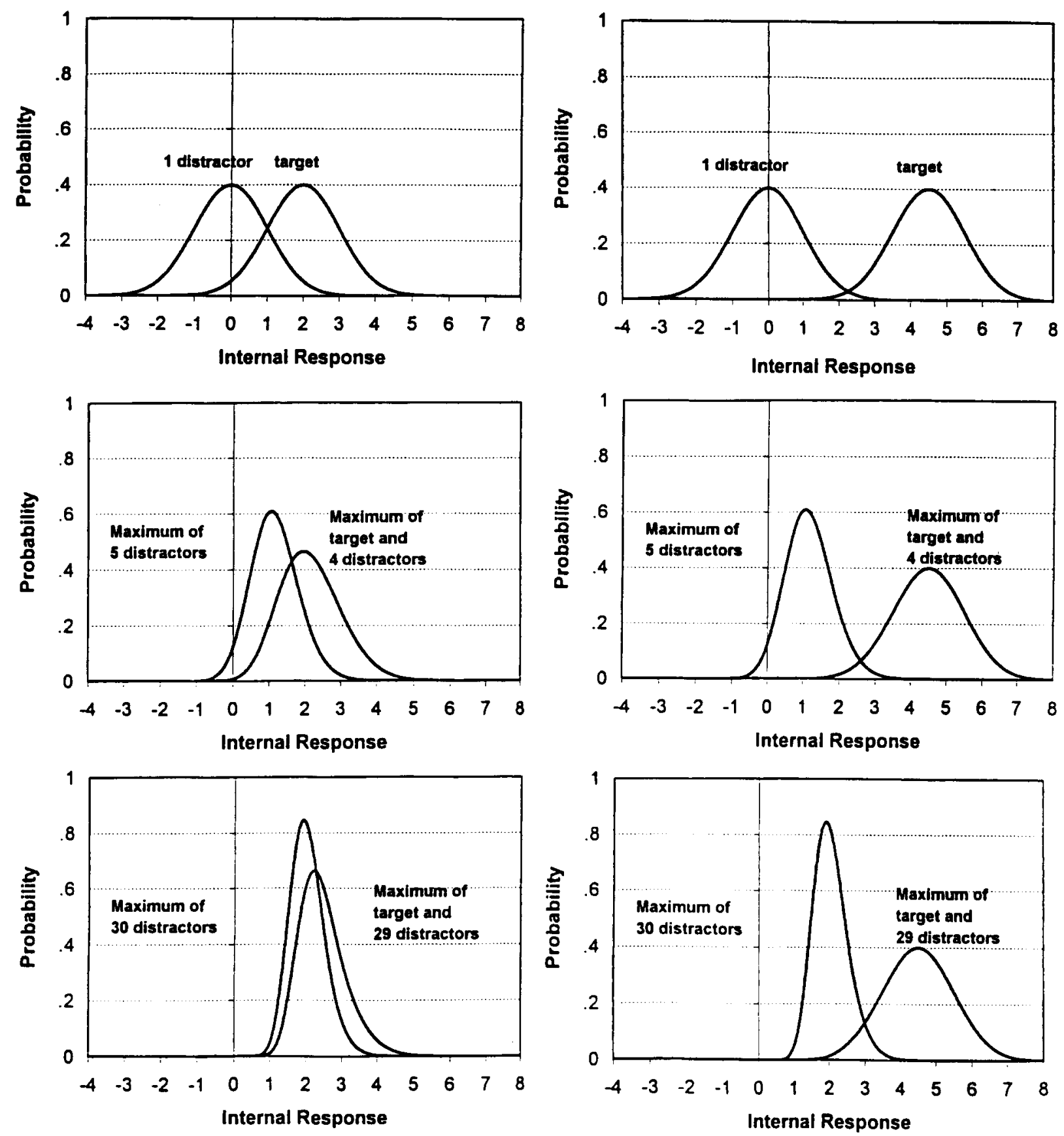

Figure 3. Probability distributions for the maximum response of the target-present alternative and maximum response of the target-absent alternative for a feature display. Overlap between the two distributions is a graphic measure of discriminability between the two alternatives. The distributions correspond to a $d_{0}^{\prime}=2.0$ for $n=1, n=5$, and $n=31$. As the number of distractors increases, the overlapping between the two distributions increases. The left panels correspond to medium target/distractor discriminability $\left(d_{0}^{\prime}=\mathbf{2 . 0}\right)$, and the right panels to high target/distractor discriminability $\left(d_{0}^{\prime}=4.5\right)$.

rule uses a linear strategy to combine the $f$ feature responses for each element into a single response, $Z_{i}$ :

$$
Z_{i}=\sum_{j=1}^{f} w_{j} \cdot x_{i j},
$$

where $Z_{i}$ is the result of combining responses along all $f$ feature dimensions for the $i$ th element, $x_{i j}$ is the internal responses along the $j$ th independent feature dimensions for the $i$ th element in the display, and $w_{j}$ is the weight applied by the observer to the $j$ th feature dimension when combining information across feature dimensions.

If we assume that the responses along the individual $f$ feature dimensions are stochastically independent and Gaussian distributed, it can be shown (Appendix B) that the effective target discriminability between the target 


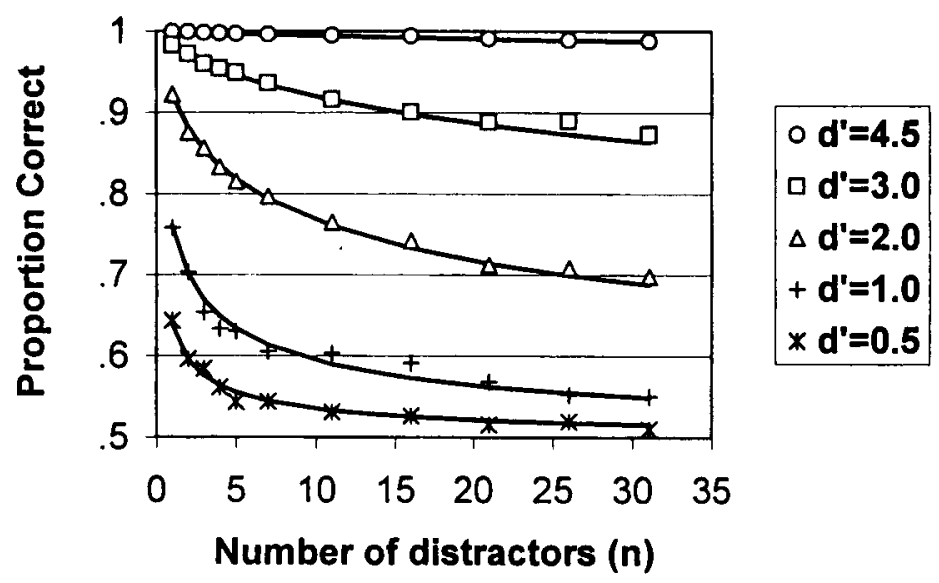

Figure 4. Proportion of correct identification of the target-present alternative in a two-alternative forced choice task as a function of number of distractors and target/distractor discriminability $\left(d_{\mathrm{o}}^{\prime}\right)$. Performance degradation as a function of number of distractors increases with lower target/ distractor discriminability over a large range of performance levels. Lines correspond to theoretical results based on numerical evaluation of equations. Symbols correspond to theoretical results based on Monte Carlo simulations.

and the $i$ th distractor, after combining information across feature dimensions, is given by

$$
d_{i z}^{\prime}=\frac{\sum_{j=1}^{f} w_{j} d_{i j}}{\sqrt{\sum_{j=1}^{f} w_{j}^{2}}},
$$

where $d_{i z}^{\prime}$ is the discriminability between the target and the $i$ th distractor type along the new decision variable $z$, $d_{i j}^{\prime}$ is the discriminability between the target and the $i$ th distractor along the $j$ th feature dimension, and $w_{j}$ is the weight used for the $j$ th feature dimension for the linear combination of information across feature dimensions.

Max-min decision rule. An observer who uses the max-min decision rule first chooses for each element in the display the feature dimension that elicits the smallest response and then chooses the alternative that contains the maximum among these responses (Figure 6). The purpose of this minimum operation is to choose for each distractor the feature dimension that has the highest target/

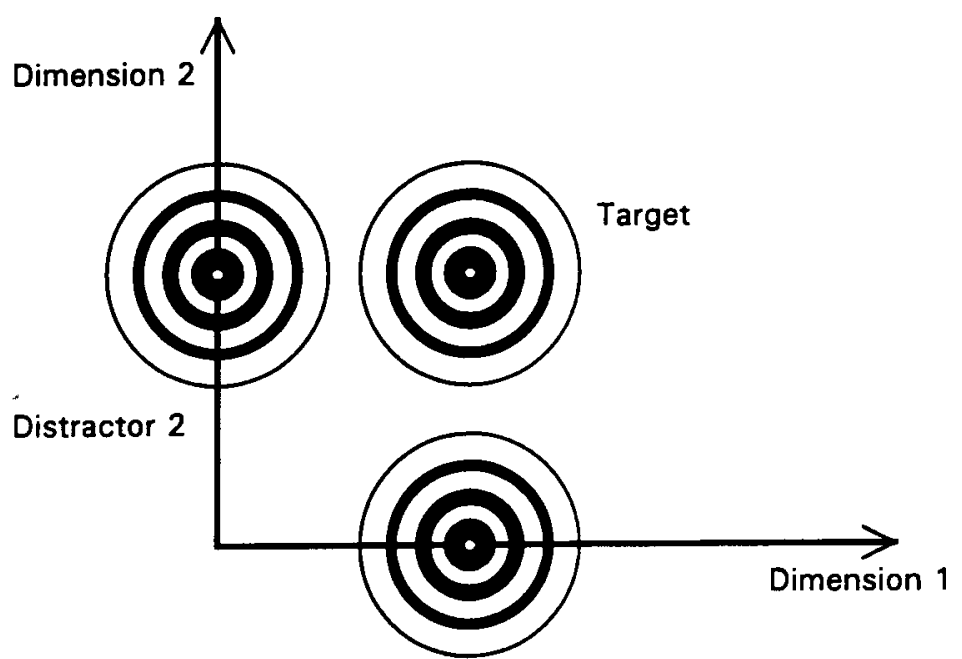

Distractor 1

Figure 5. Two-dimensional (2-D) representation of a noisy response along two independent dimensions. Concentric circles represent 2-D Gaussian distributions in which the central areas have a higher probability of occurrence than do the peripheral areas. The distributions are centered on the mean values along both dimensions. The shown graph representation corresponds to a typical conjunction display in which the target differs from each distractor along one feature dimension. Target distribution has a high mean response value along both feature dimensions. The distractor distributions have high mean response values along only one feature dimension. 
Table 1

Proportion Correct for a Feature Display as a Function of Number of Distractors for Different Levels of Target/Distractor Discriminability $\left(d_{0}^{\prime}\right)$

\begin{tabular}{|c|c|c|c|c|c|c|c|c|c|c|c|c|c|}
\hline \multirow[b]{2}{*}{$d_{\mathrm{o}}^{\prime}$} & \multicolumn{13}{|c|}{ Number of Distractors } \\
\hline & 1 & 2 & 3 & 4 & 5 & 6 & 7 & 9 & 11 & 16 & 21 & 26 & 31 \\
\hline .1 & .5282 & 5176 & .5132 & .5107 & .5090 & .5079 & .5070 & .5057 & .5049 & .5036 & .5029 & .5024 & .5021 \\
\hline .2 & .5562 & .5361 & .5274 & .5224 & .5190 & .5167 & .5149 & .5123 & .5106 & .5079 & .5064 & .5054 & .5047 \\
\hline .3 & .5840 & .5553 & .5425 & $.5351 \cdots$ & .5301 & .5265 & .5237 & .5198 & .5171 & .5129 & .5105 & .5089 & .5077 \\
\hline .4 & .6114 & .5751 & .5586 & .5487 & .5421 & .5372 & .5335 & .5282 & .5244 & .5186 & .5152 & .5130 & .5114 \\
\hline .5 & .6382 & .5955 & .5755 & .5634 & $.555 \mathrm{I}$ & .5490 & .5443 & .5375 & .5327 & .5252 & .5208 & .5178 & .5156 \\
\hline .6 & .6643 & .6164 & .5932 & .5790 & .5691 & .5618 & .5562 & .5479 & .5420 & .5327 & .5271 & .5234 & .5206 \\
\hline .7 & .6897 & .6375 & .6116 & .5954 & .5841 & .5756 & .5690 & .5592 & .5523 & .5411 & .5344 & .5298 & .5264 \\
\hline .8 & .7142 & .6588 & .6305 & .6126 & .5999 & .5903 & .5828 & .5716 & .5636 & .5506 & .5426 & .5371 & .5331 \\
\hline .9 & .7377 & .6801 & .6499 & .6304 & .6165 & .6060 & .5976 & .5850 & .5759 & .5610 & .5517 & .5454 & .5406 \\
\hline 1.0 & .7602 & .7014 & .6696 & .6488 & .6339 & .6224 & .6132 & .5993 & .5892 & .5724 & .5619 & .5546 & .5491 \\
\hline 1.1 & .7817 & .7223 & .6895 & .6677 & .6518 & .6395 & .6296 & .6145 & .6034 & .5849 & .5731 & .5649 & .5586 \\
\hline 1.2 & .8019 & .7429 & .7094 & .6869 & .6702 & .6572 & .6467 & .6306 & .6186 & .5983 & .5854 & .5762 & .5692 \\
\hline 1.3 & .8210 & .7630 & .7293 & .7062 & .6890 & .6754 & .6644 & .6474 & .6346 & .6128 & .5986 & .5885 & .5808 \\
\hline 1.4 & .8389 & .7825 & .7489 & .7255 & .7080 & .6940 & .6826 & .6648 & .6513 & .6281 & .6128 & .6018 & .5934 \\
\hline 1.5 & .8556 & .8012 & .7681 & .7448 & .7270 & .7128 & .7011 & .6827 & .6687 & .6442 & .6279 & .6161 & .6070 \\
\hline 1.6 & .8711 & .8192 & .7869 & .7638 & .7460 & .7317 & .7198 & .7010 & .6865 & .6610 & .6439 & .6313 & .6215 \\
\hline 1.7 & .8853 & .8363 & .8051 & .7824 & .7648 & .7505 & .7386 & .7196 & .7048 & .6785 & .6606 & .6474 & .6370 \\
\hline 1.8 & .8985 & .8525 & .8225 & .8005 & .7833 & .7692 & .7573 & .7382 & .7232 & .6964 & .6779 & .6641 & .6533 \\
\hline 1.9 & .9104 & .8677 & .8392 & .8181 & .8013 & .7875 & .7758 & .7568 & .7418 & .7146 & .6957 & .6815 & .6702 \\
\hline 2.0 & .9213 & .8819 & .8551 & 8349 & .8187 & .8053 & .7938 & .7752 & .7603 & .7331 & .7139 & .6994 & 6878 \\
\hline 2.1 & .9312 & .8950 & .8700 & .8509 & .8354 & .8225 & .8115 & .7932 & .7786 & .7516 & .7324 & .7176 & .7058 \\
\hline 2.2 & .9401 & .9072 & .8840 & .8660 & .8514 & .8391 & .8284 & .8108 & .7966 & .7700 & .7508 & .7361 & .7241 \\
\hline 2.3 & .9481 & .9183 & .8970 & .8802 & .8665 & 8548 & .8447 & .8279 & .8141 & .7881 & .7693 & .7546 & .7426 \\
\hline 2.4 & .9552 & .9285 & .9090 & .8935 & .8807 & .8698 & .8602 & .8442 & .8310 & .8059 & .7874 & .7729 & .7611 \\
\hline 2.5 & .9615 & .9377 & .9200 & .9058 & .8940 & .8838 & .8749 & .8597 & .8472 & .8231 & .8052 & .7911 & .7794 \\
\hline 2.6 & .9670 & .9460 & .9300 & .9172 & .9063 & .8969 & .8886 & .8744 & .8626 & .8397 & .8225 & .8088 & .7974 \\
\hline 2.7 & .9719 & .9534 & .9391 & .9275 & .9176 & .9090 & .9013 & .8882 & .8772 & .8556 & .8392 & .8260 & .8150 \\
\hline 2.8 & .9761 & .9600 & .9473 & .9369 & .9280 & .9201 & .9131 & .9010 & .8908 & .8706 & .8551 & .8426 & .8321 \\
\hline 2.9 & .9798 & .9658 & .9547 & .9454 & .9374 & .9303 & .9239 & .9129 & .9035 & .8848 & .8702 & .8584 & .8484 \\
\hline 3.0 & .9831 & .9709 & .9612 & .9530 & .9458 & .9395 & .9338 & .9238 & .9152 & .8980 & .8845 & .8734 & .8640 \\
\hline 3.1 & .9858 & .9754 & .9669 & .9597 & .9534 & .9478 & .9427 & .9337 & .9259 & .9102 & .8977 & .8874 & .8786 \\
\hline 3.2 & .9882 & .9793 & .9720 & .9657 & .9602 & .9552 & .9507 & .9426 & .9357 & .9214 & .9100 & .9005 & .8924 \\
\hline 3.3 & .9902 & .9827 & .9764 & .9709 & .9661 & .9618 & .9578 & .9507 & .9445 & .9316 & .9213 & .9126 & .9052 \\
\hline 3.4 & .9919 & .9856 & .9802 & .9755 & .9713 & .9676 & .9641 & .9578 & .9523 & .9409 & .9316 & .9238 & .9169 \\
\hline 3.5 & .9933 & .9880 & .9835 & .9795 & .9759 & .9726 & .9696 & .9642 & .9593 & .9492 & .9409 & 9339 & .9277 \\
\hline 3.6 & .9945 & .9901 & .9863 & .9829 & .9798 & .9770 & .9744 & .9697 & .9655 & .9566 & .9493 & .9430 & .9375 \\
\hline 3.7 & .9956 & .9919 & .9887 & .9858 & .9832 & .9808 & .9786 & .9746 & .9709 & .9632 & .9567 & .9512 & .9462 \\
\hline 3.8 & .9964 & .9934 & .9907 & .9883 & .9861 & .9841 & .9822 & .9788 & .9757 & .9689 & .9633 & .9584 & .9541 \\
\hline 3.9 & .9971 & .9946 & .9924 & .9904 & .9886 & .9869 & .9853 & .9824 & .9797 & .9740 & .9691 & .9648 & .9610 \\
\hline 4.0 & .9977 & .9956 & .9938 & 9922 & .9907 & .9893 & .9879 & .9855 & .9832 & .9783 & .9741 & .9704 & .9671 \\
\hline 4.1 & .9981 & .9965 & .9950 & .9937 & .9924 & .9912 & .9901 & .9881 & .9862 & .9820 & .9785 & .9753 & .9725 \\
\hline 4.2 & .9985 & .9972 & .9960 & .9949 & .9939 & .9929 & .9920 & .9903 & .9887 & .9852 & .9822 & .9795 & .9771 \\
\hline 4.3 & .9988 & .9978 & .9968 & .9959 & .9951 & 9943 & .9935 & .9921 & .9908 & .9879 & .9854 & .9831 & .9810 \\
\hline 4.4 & .9991 & .9982 & .9975 & .9967 & .9961 & .9954 & .9948 & .9936 & .9926 & .9902 & 9881 & .9861 & .9844 \\
\hline 4.5 & .9993 & .9986 & .9980 & .9974 & .9969 & .9963 & .9958 & .9949 & .9940 & .9921 & .9903 & .9887 & .9873 \\
\hline 4.6 & .9994 & .9989 & .9984 & 9980 & .9975 & .9971 & .9967 & .9959 & .9952 & .9936 & .9922 & .9909 & .9897 \\
\hline 4.7 & .9996 & .9991 & .9988 & .9984 & .9980 & .9977 & .9974 & .9968 & .9962 & .9949 & .9937 & .9927 & .9917 \\
\hline 4.8 & .9997 & .9993 & .9990 & .9987 & .9985 & .9982 & .9980 & .9975 & .9970 & .9960 & .9950 & .9941 & .9933 \\
\hline 4.9 & .9997 & .9995 & .9993 & 9990 & .9988 & .9986 & .9984 & .9980 & .9977 & .9968 & .9960 & .9953 & .9947 \\
\hline
\end{tabular}

distractor discriminability. ${ }^{2}$ In order to obtain a general expression for the performance of the max-min observer, we need to first obtain the probability distribution of the minimum response among $f$ feature dimensions. The distribution of the minimum response among $f$ feature dimension responses for each individual element is given by

$$
\begin{aligned}
e_{i, \min }(x) & =\operatorname{Min}\left\{x_{i j}, j=1, \ldots, f\right\} \\
& =\sum_{j=1}^{f} e_{i j}(x) \cdot \prod_{k=1}^{f}\left[E_{i k}(>x)\right]^{\left(1-\delta_{j k}\right)},
\end{aligned}
$$

where $e_{i, \min }(x)$ is the probability that the minimum among the $i$ th element's $f$ feature responses will take a value $x, e_{i j}(x)$ is the probability of the $i$ th element's eliciting a response of value $x$, along the $j$ th feature dimension, $E_{i k}(>x)$ is the cumulative probability of the $i$ th element's eliciting a value greater than $x$ along the $k$ th feature dimension, and $\delta_{j k}$ is the Kronecker delta, which is 1 for $j=k$ and 0 otherwise. Equation 5 can be used to calculate, for each element (target and different distractors), the probability functions for the minimum response among all the individual feature responses. These minimum responses are used as the decision variable, and 


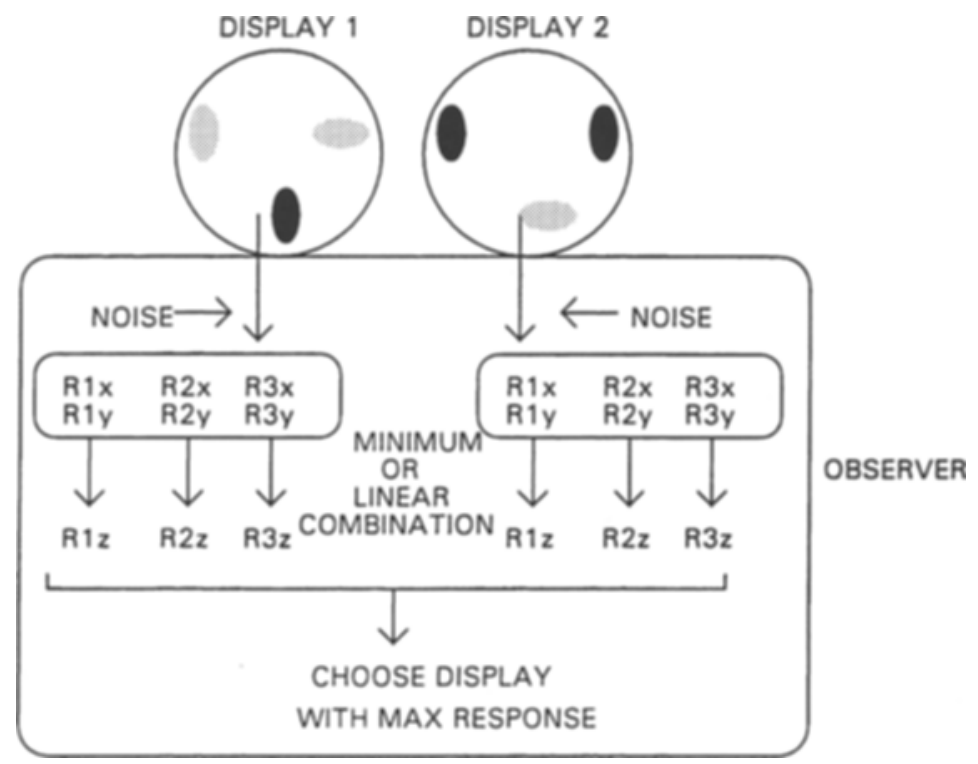

Figure 6. Max-linear and max-min decision rules for a conjunction of contrast and orientation search task for a two-alternative forced choice. The target is a vertical high-contrast ellipse among vertical low-contrast ellipses and a horizontal high-contrast ellipse. The observers' task is to correctly identify the alternative with the target. The observer is assumed to monitor two independent noisy responses per element, corresponding to the two feature dimensions. For each element, the two responses are combined through a linear combination rule or a minimum rule, resulting in a single decision variable. The observer then chooses the alternative eliciting the highest response along the decision variable.

the observer is assumed to choose the response alternative containing the maximum response among all the elements' minimum feature responses.

\section{Multiple Distractor Types Along the}

New Decision Variable (Distractor Variability)

If there are different types of distractors in the display, the target/distractor discriminability along the new decision variable, after combining information across feature dimensions, might be different for different distractors. For the case in which there are different distractors with different associated target/distractor discriminabilities along the new decision variable, we assume that different distractors elicit noisy values with different means.

For the case of multiple distractors, the decision rule remains the same as the case with a single distractor type, where the observer monitors all internal responses in the target-present and target-absent displays and selects on each trial the display containing the maximum response. The $P_{\mathrm{c}}$ in identifying the target-present display for the case of multiple distractor types can be calculated by computing the probability that the maximum response in the target-present display will exceed the maximum response in the target-absent display. A general purpose mathematical expression can be derived to predict $P_{\mathrm{c}}$ for a given target, a set of distractors $\left(N=\left\{n_{1}, n_{2}, n_{3}, \ldots\right.\right.$, $\left.n_{\mathrm{k}}\right\}$, with $k$ different distractor types having $n_{i}$ number of distractors), and number of response alternatives $M$ (see Appendix $\mathrm{C}$ for derivation). ${ }^{3}$

\section{SIMPLIFIED FORMULATION FOR MULTIDIMENSIONAL SEARCH DISPLAYS}

In the previous two sections, we developed the general framework of SDT for multidimensional displays. However, with the additional assumption that the target/distractor discriminabilities along the different individual feature dimensions are approximately equal, the predictions of the SDT model for multidimensional displays are greatly simplified.

\section{Combining Information}

\section{Across Feature Dimensions}

Max-linear decision rule. For the case in which the internal target/distractor discriminability is approximately the same across the different feature dimensions, ${ }^{4}$ one might assume that the observer might equally weight information across different dimensions (averaging or straight summation). For this special case, a simple rule (feature combination rule) can be used to calculate the effective target/distractor discriminability (see Appendix $B$ for derivation).

The feature combination rule states that, if $f$ is the number of relevant feature dimensions across which the 
internal responses are combined, $r$ is the number of feature dimensions along which the distractor and the target differ, and $d_{\mathrm{o}}^{\prime}$ the target/distractor discriminability along each of the the $r$ feature dimensions, the effective target/ distractor discriminability is given by

$$
d_{r, f}^{\prime}=\frac{r d_{\mathrm{o}}^{\prime}}{\sqrt{f}} .
$$

The probability function for the target, after combination across feature dimensions, is then given by $t_{f}(x)=g(x-$ $\left.d^{\prime}{ }_{r, f}\right)$, and that for the distractor is given by $d_{r, f}(x)=g(x)$.

Max-min decision rule. For the special case in which the target/distractor similarity is the same along the individual feature dimensions and the individual internal responses are Gaussian distributed, the target probability function for the max-min observer is given by

$$
t_{\min f}(x)=f g\left(x-d_{\mathrm{o}}^{\prime}\right) G\left(>x-d_{\mathrm{o}}^{\prime}\right)^{f-1},
$$

where the notation is as previously defined.

Similarly, for the distractors, we obtain

$$
\begin{aligned}
d_{\min r, f}(x)= & (f-r) g\left(x-d_{\mathrm{o}}^{\prime}\right) G\left(>x-d_{\mathrm{o}}^{\prime}\right)^{f^{-r-1} G(>x)^{r}} \\
& +r g(x) G(>x)^{r-1} G\left(>x-d_{\mathrm{o}}^{\prime}\right)^{f^{-r}}
\end{aligned}
$$

\section{APPLICATION TO COMMON DESIGNS}

\section{Conjunction Displays}

In the typical conjunction search task (Treisman, 1991; Treisman \& Gelade, 1980), half the distractors are different from the target along a dimension or stimulus attribute $x_{1}$, and the other half of the distractors are different from the target along another dimension or stimulus attribute $x_{2}$. For example, Figure 7 (row 2, column 1) shows a possible task in which the two relevant dimensions are contrast and orientation. The target is a vertical high-contrast ellipse. Half the distractors are horizontally oriented and have high contrast, and the other half of the distractors are vertically oriented and have low contrast. In order to generate quantitative predictions for the SDT model for conjunction displays, we apply the two decision rules (max-linear and max-min).

Max-linear decision rule. For the conjunction display, the information is combined across two feature dimensions $(f=2)$, and each distractor differs from the target along one feature dimension. Using the feature combination rule (Equation 6), we find that the effective target/distractor discriminability for both distractors is given by

$$
d_{\text {conjunction }}^{\prime}=d_{r=1, f=2}^{\prime}=\frac{d_{\mathrm{o}}^{\prime}}{\sqrt{2}} \text {. }
$$

The effective target/distractor discriminability can then be used with Equation 2 to obtain $P_{\mathrm{c}}$ for increasing set size. Performance predictions in a conjunction display are identical to those in a feature display (Equation 2), except for an additional factor of $\sqrt{2}$ dividing $d_{\mathrm{o}}^{\prime}$. This observation leads to the prediction that, if $P_{\mathrm{c}}$ in a conjunction display is transformed to a $d^{\prime}$ value, using Equa- tion 2, the max-linear model predicts a $d^{\prime}$ that is lower by a factor of $\sqrt{2}$ than the $d^{\prime}$ found for performance for the corresponding feature display (where the target/distractor discriminability along the feature dimension is kept the same as that for the conjunction display). This prediction holds for all levels of target/distractor discriminability $\left(d_{0}^{\prime}\right)$ and number of distractors $(n)$.

Max-min decision rule. The purpose of the initial minimum operation among feature dimensions in the $\max -\min$ decision rule is to select the feature dimension in the distractors that differs from the target the most. Since for the conjunction displays, the target has a higher mean response along both dimensions and the distractors have a higher mean along one dimension, the minimum operation will tend to select in the distractors the dimension that has the lowest mean response (and, therefore, the dimension that differs in mean response from the target).

The maximum operation will then tend to select the target over the distractors. In order to obtain an expression for the max-min decision rule for conjunction displays, we replace the $f=2$ and $r=1$ in Equations 7 and 8 to obtain the probability functions for the minimum among $f$ feature responses:

$$
t_{\min f=2}(x)=2 g\left(x-d_{\mathrm{o}}^{\prime}\right) \cdot G\left(>x-d_{\mathrm{o}}^{\prime}\right)
$$

and

$$
\begin{aligned}
d_{\min r=1, f=2}(x)= & g\left(x-d_{\mathrm{o}}^{\prime}\right) \\
& \cdot G(>x)+g(x) \cdot G\left(>x-d_{\mathrm{o}}^{\prime}\right) .
\end{aligned}
$$

Replacing these expressions into Equation 1, one can obtain an expression for $P_{\mathrm{c}}$ as a function of number of distractors $(n)$, number of response alternatives $(M)$, and internal target/distractor discriminability $\left(d_{0}^{\prime}\right)$.

Theoretical results. Figure 8 shows that, for both models for a given level of target/distractor discriminability $\left(d_{0}^{\prime}\right)$ along an individual feature dimension, overall performance is reduced in a conjunction versus a feature display. In the present comparison, the feature and conjunction displays have the same target/distractor difference along one feature dimension, and in the conjunction display, the target/distractor internal discriminability has been matched along both feature dimensions (Treisman, 1991). The results show that degradation as a function of number of distractors increases in the conjunction condition for both models (Figure 8), although the increase in performance degradation is larger for the max-linear model.

Discussion. Our theoretical results show that the SDT model predicts larger set-size effects for conjunction displays than for feature displays for any given level of target/distractor discriminability $\left(d_{\mathrm{o}}^{\prime}\right)$. In the SDT model, the lower search efficiency in conjunction displays is a consequence of the combination of noisy activity across the two independent feature dimensions, given the specific task configuration. Since, for each type of distractor, there is only one feature dimension that provides information by which to discriminate it from the target, the 


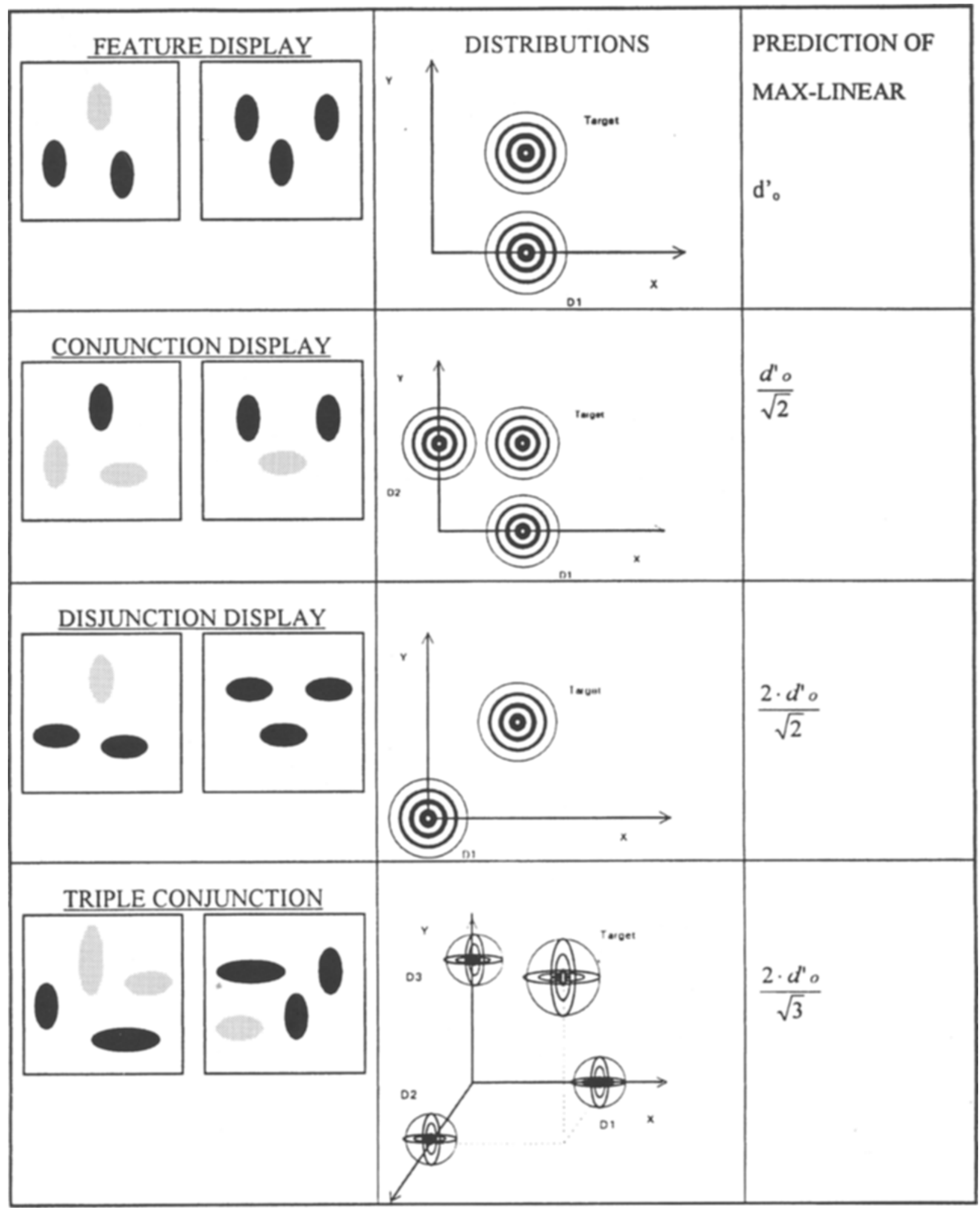

Figure 7. Summary of definitions, properties, and predictions of the search displays investigated in this paper. From top to bottom: feature, conjunction, disjunction, and triple conjunction displays. The left panels show examples of the corresponding search displays. The middle panels show two-dimensional (2-D) representations of the noisy internal responses for target and distractors for the different search displays. The concentric circles represent 2-D Gaussian distributions. The right panels are the performance predictions for the max-linear observers for the different displays, given that the experimentally obtained proportions correct are transformed to $d^{\prime}$ using Equation 2. Predictions for the max-min observers are more complex and are not summarized in the diagram. 


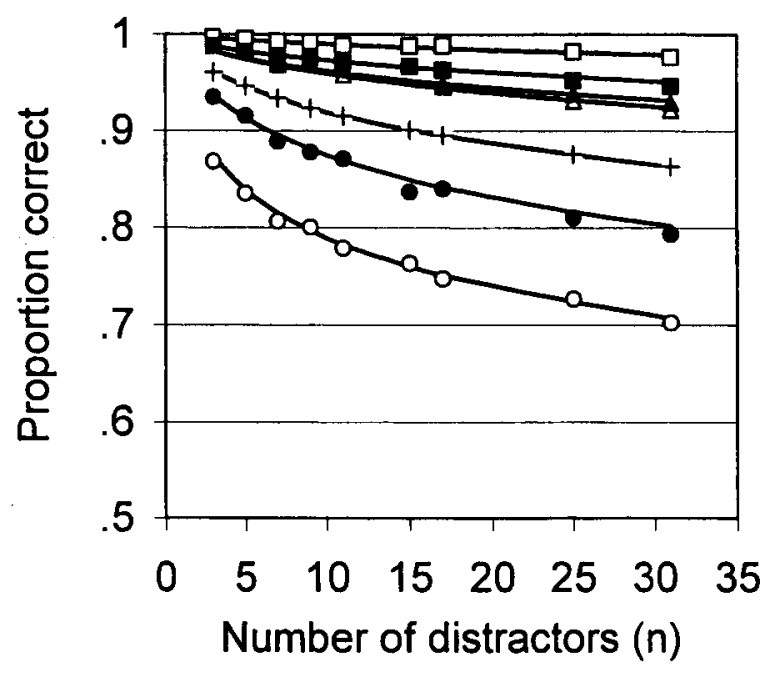

$\square$ Disjunction ML

- Disjunction MM

$\triangle$ Triple Conj MM

$\triangle$ Triple Conj ML

+Feature

- Conjunction MM

o Conjunction ML

\begin{abstract}
Figure 8. Performance (proportion correct) in identifying the alternative containing the target for the max-linear (ML) observer and the max-min (MM) observer in a twoalternative forced choice task as a function of number of distractors for feature, conjunction, triple conjunction, and disjunction displays for $d_{0}^{\prime}=3.0$. Lines correspond to theoretical results based on numerical evaluation of equations. Symbols correspond to theoretical results based on Monte Carlo simulations.
\end{abstract}

effect of combining information across an informative noisy encoder and another, noninformative noisy encoder is to reduce the discriminability between target and distractors along the new decision variable. The reduction in the effective target/distractor discriminability along the new decision variable is what causes the conjunction displays to have larger set-size effects.

\section{Triple Conjunctions}

Wolfe, Cave, and Franzel (1989) used a display that included three types of distractors and each three distractors shared a different feature with the target (see, e.g., row 4, column 1 in Figure 7, where the target is a large vertical high-contrast ellipse and the distractors are a small vertical low-contrast ellipse, a small diagonal highcontrast ellipse, and a large diagonal low-contrast ellipse). He called this display a triple conjunction display. Results showed that set-size effects in search time studies were larger for regular conjunctions than for triple conjunctions. This finding was not predicted by standard feature integration theory and led to a modification of that model (guided search model; Wolfe, 1994; Wolfe et al., 1989 ) in order to account for the findings.

We will develop the predictions of the SDT model and compare them with simple conjunctions and feature displays for the case in which the internal target/distractor discriminability $\left(d_{\mathrm{o}}^{\prime}\right)$ is matched across the three feature dimensions and is kept constant across displays.

Max-linear decision rule. For triple conjunctions, information is combined across three feature dimensions $(f=3)$, and each distractor differs from the target along two feature dimensions $(r=2)$. Using Equation 6 , we then obtain

$$
d_{\text {triple conjunction }}^{\prime}=d_{r=2, f=3}^{\prime}=\frac{2 d_{\mathrm{o}}^{\prime}}{\sqrt{3}} .
$$

The prediction of the SDT model with a max-linear decision rule is that, if performance is obtained in a triple conjunction display and converted to $d^{\prime}$, using Equation 2 , the resulting $d^{\prime}$ will be larger by a factor of $2 / \sqrt{3}$ than that obtained for the corresponding feature condition (along any of the three feature dimensions in the task). Figure 7 summarizes the model predictions for the maxlinear model in a triple conjunction display.

Max-min decision rule. Since the target has a high mean along three feature dimensions and the distractors have a high mean along one feature dimension, the minimum operation will tend to select in the distractors one of the two feature dimensions with the lowest mean. The maximum operation will tend to select the target over the distractors. In order to obtain an expression for the max-min decision rule for conjunction displays, we replace the $f=3$ and $r=2$ in Equations 7 and 8 to obtain the probability functions for the minimum among $f$ feature responses:

$$
t_{\min f=3}(x)=3 \cdot g\left(x-d_{\mathrm{o}}^{\prime}\right) \cdot G^{2}\left(>x-d_{\mathrm{o}}^{\prime}\right)
$$

and

$$
\begin{aligned}
d_{\min r=2, f=3}(x)= & g\left(x-d_{\mathrm{o}}^{\prime}\right) \cdot G^{2}(>x)+2 \\
& \cdot g(x) \cdot G(x) \cdot G\left(>x-d_{\mathrm{o}}^{\prime}\right) .
\end{aligned}
$$

Replacing the probability functions for the target and the distractor in Equation 1, one can obtain an expression for $P_{\mathrm{c}}$ for triple conjunctions.

Theoretical results. Comparison of the results in Figure 8 shows that performance for the max-min model is 
slightly better than that for the max-linear model. Figure 8 shows that, for both models at a given level of target/ distractor discriminability $\left(d_{\mathrm{o}}^{\prime}\right)$ along an individual feature dimension, overall performance is higher for the triple conjunction than for the conjunction and feature displays. In the present comparison, the target/distractor physical differences for the conjunction and triple conjunction displays are matched.

Discussion. In the SDT formulation, the improvement in performance from the conjunction display to the triple conjunction display is due to the fact that the target differs from each distractor along two feature dimensions. The effect of combining information across two informative and a noninformative noisy feature responses is a noisier decision variable (by a factor of $\sqrt{3}$ ), but one that discriminates target responses from distractor responses better (by a factor of 2). In this way, SDT predicts higher set-size effects for conjunctions versus triple conjunctions without resorting to a two-stage model consisting of a parallel stage guiding a serial processor (Wolfe et al., 1989).

\section{Disjunction (Two-Dimensional Feature)}

Treisman (1991) measured search response times for a display in which the target differed from the distractors along two feature dimensions (disjunctions). In order to match distractor variability with respect to conjunction displays, two distractors were used in the disjunction display. The results showed that, even when target/distractor physical difference and distractor variability are matched across displays, disjunctions result in smaller set-size effects than do conjunction displays. In this section, we apply SDT to a disjunction display similar to that used by Treisman. Unlike Treisman's disjunction display, which consisted of two type of distractors, we generated model predictions for a disjunction display with one type of distractor. However, our results could be easily generalized to any number of distractor types. Figure 7 (row 3, column 1) shows the disjunction display in which the target differs from the distractor along two feature dimensions.

Max-linear decision rule. Noting that, for the case of our disjunction displays, information is combined across two feature dimensions $(f=2)$ and that the target differs from the distractor along the two feature dimensions $(r=2)$, we obtain from Equation 6

$$
d_{\text {disjunction }}^{\prime}=d_{r=2, f=2}^{\prime}=\frac{2 d_{\mathrm{o}}^{\prime}}{\sqrt{2}} \text {. }
$$

The equation reduces to an expression that is identical to the equation describing the feature search, except for the fact that a $d_{\mathrm{o}}^{\prime}$ is multiplied by a factor of $2 / \sqrt{2}$ (target/ distractor discriminability along the individual feature dimensions). The prediction of the SDT model with the max-linear decision rule is that, if performance in a disjunction display is converted to $d^{\prime}$, using Equation 2 , the resulting $d^{\prime}$ will be larger by a factor of $2 / \sqrt{2}$ than that obtained for the corresponding feature condition (along any of the two feature dimensions in the task).
Max-min decision rule. Since the target has a high mean response along both dimensions and the distractors have a low mean response along both dimensions, the minimum operation will tend to select any of the two target and distractor feature dimensions. The maximum operation will then tend to select the target response over the distractors. ${ }^{5}$ In order to obtain an expression for the max-min decision rule for disjunction displays, we use Equations 7 and 8 to obtain

$$
t_{\min f=2}(x)=2 \cdot g\left(x-d_{\mathrm{o}}^{\prime}\right) \cdot G\left(>x-d_{\mathrm{o}}^{\prime}\right)
$$

and

$$
d_{\min r=2, f=2}(x)=2 \cdot g(x) \cdot G(>x) .
$$

Replacing the probability functions for target and distractor into Equation 1, one can obtain an expression for $P_{\mathrm{c}}$ as a function of number of distractors $(n)$ for disjunction displays.

Theoretical results. Figure 8 shows that performance in disjunction displays for the max-linear model is somewhat superior than that for the max-min model (the opposite of what was found for conjunction displays) for a fixed level of target distractor discriminability $\left(d_{\mathrm{o}}^{\prime}\right)$ and number of distractors $(n)$. Figure 8 shows that, for both models for a given level of target/distractor discriminability $\left(d_{0}^{\prime}\right)$ along an individual feature dimension, overall performance is higher for the disjunction displays than for the triple conjunction, feature, and conjunction displays. For the present comparison, the target/distractor physical differences are kept constant across displays.

Discussion. Although our results agree qualitatively with the finding in search time studies of a higher search efficiency for disjunction than for conjunction displays (Treisman, 1991), our particular disjunction display is not identical to the one used for Treisman's search time study, where the target was a vertical blue bar, half the distractors were right oriented $27^{\circ}$ and violet, and half the distractors were left oriented $27^{\circ}$ and turquoise. A main difference is that, in Treisman's display, the target's value lies in between the mean values of the distractors (along the hue and orientation dimensions). Although the maxlinear decision rule does not apply directly to this display, it can be shown that, with a change of variables, the two displays become identical and that the max-linear model makes identical predictions for the disjunction display addressed in this paper and the disjunction display investigated by Treisman. ${ }^{6}$ In summary, the SDT model predicts performance improvement from conjunction to disjunction displays.

\section{Matching Performance Across Display Types}

The SDT predictions in the previous sections correspond to displays in which the physical difference between target and distractors along the different dimensions are matched across displays (feature vs. conjunction, triple conjunction, and disjunction). However, what if the target/distractor discriminability along the individ- 


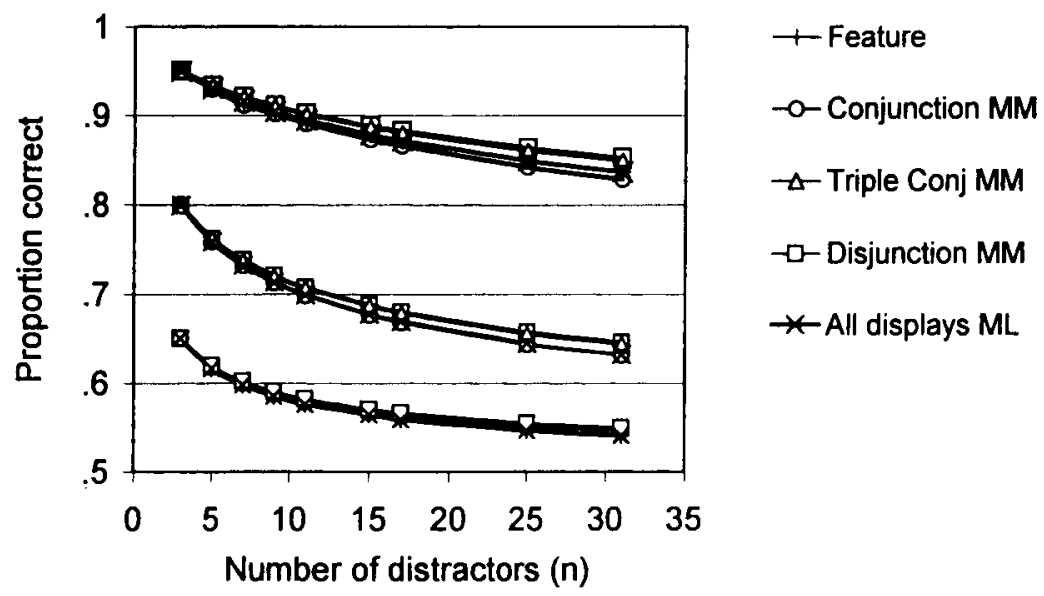

Figure 9. Performance (proportion correct) in identifying the alternative containing the target for the max-min (MM) and the max-linear (ML) observer in a twoalternative forced choice task as a function of number of distractors for the case where performance was matched across displays for the case of $n=3$. The graph shows feature, conjunction, triple conjunction, and disjunction displays. Lines correspond to theoretical results based on numerical evaluation of equations. Symbols correspond to theoretical results based on Monte Carlo simulations.

ual feature dimensions in the multidimensional display is increased so that $P_{\mathrm{c}}$ in the $n=2$ condition in the feature and the multidimensional display (e.g., conjunction, disjunction, triple conjunction) are equal? ${ }^{7}$

Figure 9 shows that, if the target/distractor internal discriminability $\left(d_{0}^{\prime}\right)$ along the individual feature dimensions are adjusted so that performance across both display types are matched for a given set size $(n)$, the set-size effects for the max-linear observer become the same for all three display conditions and approximately the same as those for the max-min observer.

\section{The Effect of Target/Distractor \\ Discriminability in Multidimensional Displays}

Manipulating the target/distractor discriminability along each individual feature dimension will also affect performance in multidimensional displays. For the maxlinear model and the specific displays investigated in this paper (where $d_{r, f}^{\prime}$, the target/distractor discriminability, along all $r$ feature dimensions is equal), the effect of changing the target/distractor discriminability $\left(d_{r, f}^{\prime}\right)$ on $P_{\mathrm{c}}$ is the same as that for the feature task and is entirely described by Figure 4 . For the max-min observer, a similar effect is found.

\section{EXPERIMENT \\ Testing Set-Size Effects in Disjunctive and Conjunctive Displays}

To illustrate the applicability of SDT to visual search accuracy in multidimensional displays, we use human data from experiments performed at the University of Washington by Aiken and Palmer (1992). They used conjunction and disjunction displays that were somewhat different than the ones previously presented in this paper. The conjunction display had an additional third distractor that had a smaller mean response than did the target along both feature dimensions (Figure 10). The disjunction display had two additional distractors that also differed along both dimensions from the target (Figure 10). Thus, the distractors were identical in the conjunction and disjunction conditions. There were three conditions: (1) conjunction display, (2) disjunction display, and (3) disjunction display with reduced target/distractor discriminability.

For each condition, search accuracy was measured for two and eight distractors $(n=2$ and $n=8)$. We applied the developed SDT-based model in an attempt to quantitatively predict human visual search accuracy for the displays used. The predictions are compared with the predictions of a limited capacity serial mechanism previously used by Bergen and Julesz (1983a, 1983b) to predict search accuracy for a $\mathrm{T}$ among Ls. In the temporally serial model, the observer can perfectly process $h$ items per presentation time. When the display contains fewer than $h$ items, the observer performs perfectly $(100 \%)$. On the other hand, when there are more than $h$ items in the display, the observer processes $h$ random elements of the total $n$ elements in the display (without processing the same element twice). The Data Analysis section describes the details about the mathematical fit of the serial model to the data.

\section{Method}

Subjects. Three male young adults (age, 23-28 years) with normal or corrected acuity participated in the study.

Apparatus. Images were displayed on a 13-in. Apple color monitor driven by a Macintosh IIcx computer. The monitor had a background luminance of $200 \mathrm{~cd} / \mathrm{m}^{2}$ and a resolution of 640 by 480 pix- 


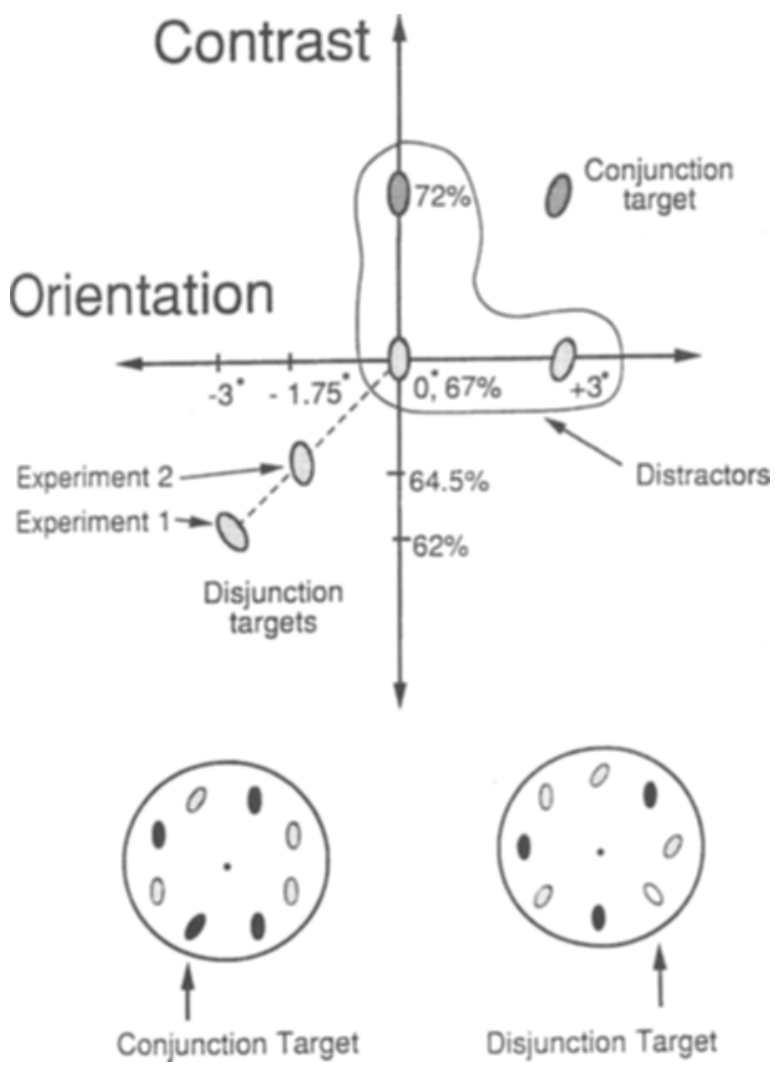

Figure 10. Modified conjunction and disjunction displays used in the Aiken and Palmer (1992) studies. The top diagram shows the contrast and orientation values for the ellipsoidal targets and distractors used in the conjunction and disjunction experiments by Aiken and Palmer. The bottom diagrams show sample displays used in the experiments, in which the elements appear along the circumference of a circle centered on a fixation point.

els. Viewing distance was from $61 \mathrm{~cm}$, resulting in a subtended angle of 2 min of arc per pixel.

Stimuli. The stimuli for the experiment were ellipses with major axes of $30 \mathrm{arc}$ min and minor axes of $10 \mathrm{arcmin}$. The ellipses lay on the perimeter of an imaginary circle with a radius of $6^{\circ}$ of arc centered on the fixation point (Figure 10). Manipulations of the stimuli were performed along two physical attributes: ellipse contrast and orientation. A pilot study was used to determine how to match changes along both dimensions in order to achieve the same search accuracy. These pilot studies revealed that, for a standard ellipse of vertical orientation and $61 \%$ contrast, a $5 \%$ change in contrast produced approximately the same accuracy as a $3^{\circ}$ change in orientation.

Two displays were investigated. In the conjunction display, the target ellipse had an orientation of $+3^{\circ}$ and a contrast of $72 \%$. There were three types of distractors: (1) distractors with $0^{\circ}$ orientation and $67 \%$ contrast, (2) distractors with $+3^{\circ}$ orientation and $67 \%$ contrast, and (3) distractors with $0^{\circ}$ orientation and $72 \%$ contrast. In this way, two of the distractors shared the same value along one of the feature dimensions with the target. The third distractor differed from the target along both feature dimensions.

In the disjunction display, the distractors remained the same, but the target was an ellipse with $62 \%$ contrast and an orientation of $-3^{\circ}$. In this way, the target differed from the distractors along both feature dimensions. A second disjunction condition was also studied, in which the target/distractor discriminability was reduced by making the target an ellipse with $64.5 \%$ contrast and an orientation of $-1.75^{\circ}$.

One additional complication of the design of the displays used by Aiken and Palmer (1992) was that the appearance of a distractor was probabilistic. Instead of assigning, from trial to trial, a fixed number of $i$ th type distractors to the displays, distractors were sampled randomly from the three distractor types with a probability of $1 / 3$. This design added the additional complication that the display could have different distractor configurations from trial to trial. For example, for the case of $n=3$, the alternative containing the target might have two distractors of Type 1, two distractors of Type 2, two distractors of Type 3, one distractor of Type 1 and one distractor of Type 2, and so on. Appendix D develops in detail the application of the SDT model to this particular design, in order to generate the quantitative predictions to be compared with the human data.

Procedure. The display was presented to the subjects in the following sequence. A fixation point was first presented for $100 \mathrm{msec}$, followed by an interstimulus interval of $1,000 \mathrm{msec}$. The first stimulus display alternative was shown for $100 \mathrm{msec}$, followed by another interstimulus interval of $1,000 \mathrm{msec}$. Finally, the second stimulus display alternative was presented for $100 \mathrm{msec}$. The observers had unlimited time in which to make their decisions.

Data analysis. $P_{c}$ for identifications of the alternative display containing the target was computed for each subject and condition. Performance for each of the possible display configurations can be calculated (Appendix D). The probability of occurrence of each possible display configuration can also be calculated, given that each distractor has a $1 / 3$ probability of being sampled. Finally, an expectation value for $P_{\mathrm{c}}$ can be obtained:

$$
E\left(P_{\mathrm{c}}\right)=p_{1} \cdot P_{\mathrm{c} 1}+p_{2} \cdot P_{\mathrm{c} 2}+p_{3} \cdot P_{\mathrm{c} 3}+\ldots+p_{k} \cdot P_{\mathrm{c} k}=\sum_{i=1}^{k} p_{i} \cdot P_{\mathrm{c} i}
$$

where $p_{i}$ is the probability of the $i$ th configuration and $P_{\mathrm{c} i}$ is the model prediction for the $i$ th distractor configuration. Equations D6 through D10 in Appendix D were used to iteratively change the value of $d_{o}^{\prime}$ to provide the best chi-square fit to the data. In a first analysis, the fits were independently done for each display type. The expected variance (denominator) in the chi-square goodness of fit was based on the statistical variance in $P_{\mathrm{c}}$ based on the binomial variance. In a second analysis, for each observer, simultaneous fits were performed for all conditions with one free parameter $d_{0}^{\prime}$.

Performance for the limited capacity serial mechanism for visual search accuracy was previously used by Bergen and Julesz (1983b). Performance as a function of the number of elements in the display $(n)$ and the number of elements that can be processed serially in the presentation time $(h)$ is given by

$$
P_{\mathrm{c}}(n, h)=0.5+\frac{h}{2 n}, \text { for } n \geq h,
$$

and

$$
P_{\mathrm{c}}(n, h)=1.0, \text { for } n<h .
$$

Equation 18 was fit to the human data for the conjunction displays, with $h$ as the only free parameter.

\section{Results}

Table 2 summarizes the measured $P_{\mathrm{c}}$ for performances for the different observers in the three different experimental conditions. Figures $11 \mathrm{~A}$ through $11 \mathrm{C}$ show simultaneous fits of the max-linear and the max-min decision rules for each observer to all three conditions. In order to make these fits, an additional assumption was made about linearity between the physical difference in 
Table 2

Proportion Correct for Three Observers for Three Display Conditions: Conjunction, Disjunction With High Target/Distractor Discriminability, and Disjunction With Low Target/Distractor Discriminability

\begin{tabular}{|c|c|c|c|c|c|c|}
\hline \multirow[b]{2}{*}{ Subject } & \multicolumn{2}{|c|}{ Conjunction } & \multicolumn{2}{|c|}{ Disjunction 1} & \multicolumn{2}{|c|}{ Disjunction 2} \\
\hline & $n=2$ & $n=8$ & $n=2$ & $n=8$ & $n=2$ & $n=8$ \\
\hline 1 & .785 & .685 & .94 & .88 & .85 & .71 \\
\hline 2 & .64 & .585 & .86 & .67 & .74 & .60 \\
\hline 3 & .70 & .605 & .96 & .86 & .78 & .64 \\
\hline
\end{tabular}

Note-Performance is for two set-size levels: $n=2$ and $n=8$.

contrast and orientation and the internal target/distractor discriminability $\left(d_{\mathrm{o}}^{\prime}\right)$ along the feature dimensions.

In order to compare the performance predictions of the max-linear model for the Aiken and Palmer (1992) modified conjunction and disjunction displays with those for the standard conjunction and disjunction displays (rows 2 and 4 of Figure 7), $P_{\mathrm{c}}$ predictions of the model were converted to $d^{\prime}$, using Equation 2 (for feature displays). As Figure 7 summarizes, the result of such a transformation for the conjunction display is a $d^{\prime}$ that is smaller by a factor of $\sqrt{2}$ than the $d^{\prime}$ for the feature display (for all levels of target/distractor discriminability and number of distractors). For the disjunction display, the transformation results in a $d^{\prime}$ that is larger by a factor of $\sqrt{2}$ than the $d^{\prime}$ for the feature display. For the modified Aiken and Palmer conjunction display, use of Equation 2 to transform $P_{\mathrm{c}}$ to $d^{\prime}$ results in a $d^{\prime}$ that is smaller by a factor of $1.25 / \sqrt{2}$ than the $d^{\prime}$ for the corresponding feature condition. For the Aiken and Palmer disjunction condition, the result of the transformation is a $d^{\prime}$ that is larger by a factor of $1.25 \cdot 2 / \sqrt{2}$ than the $d^{\prime}$ for the feature condition. Table 3 summarizes the performance predictions, using Equation 4 for the max-linear observer for a feature condition, a standard conjunction condition, a standard disjunction condition, and the Aiken and Palmer modified conjunction and disjunction conditions. The max-linear prediction of the model for the ratio between the $d^{\prime}$ 's for the conjunction and disjunction displays (using Equation 2) is 2.0 for both the standard displays (Figure 7) and the Aiken and Palmer modified conjunction and disjunction displays.

The ratios between the $d$ 's (using Equation 2) for the two display conditions for the 3 human observers were $1.78,2.25$, and 2.78 for the $n=2$ condition and 1.77, 1.7, and 2.50 for the $n=8$ condition. Averaging across conditions and observers, the ratio of $d$ 's between the conjunction and the disjunction displays is 2.13 .

Table 4 (upper part), shows the chi-square values for the simultaneous fits for 3 observers. The model could not be rejected $(p>.01)$ for Observers 1 and 2 (for both models) but was rejected for Observer 3 (for both models). For Observer 1, the max-min model provided a better fit, but for Observer 2, the max-linear model provided a better overall fit. Table 4 (lower part) shows the chi- square values for separate fits for each display condition. These fits do not make any assumptions about the relation between the physical difference in contrast and orientation and the internal target/distractor discriminability $\left(d_{0}^{\prime}\right)$. The model could not be rejected $(p>.01)$, except for the conjunction condition for Observer 2 .

Table 4 also shows the chi-square values for the best fit of the temporally serial model to the conjunction condition for each observer. Figures $11 \mathrm{~A}-11 \mathrm{C}$ also show the best fit for the limited capacity serial model for the conjunction condition for the 3 observers. For all the observers, the best fit was for $h=1$ (Equation 18), which corresponds to a processing of 10 items per sec. The limited capacity serial model could be rejected for all $3 \mathrm{ob}-$ servers $(p<.01)$.

\section{Discussion}

For Observer 1, the set-size effects were larger for the conjunction type displays than for the disjunction type displays. However, for Observer 2, the set-size effect was larger for the disjunction than for the conjunctions. This is due to the fact that Observer 2 was operating at lower performance levels (low target/distractor discriminability), where the performance is closer to the $50 \%$ floor level. At these low levels of performance, set-size effects decrease with decreasing target/distractor discriminability.

The separate fits to each condition show that the SDT model with two possible decision strategies (max-linear or $\max -\min$ ) can be used to predict performance degradation in visual search accuracy as a function of the number of distractors for conjunctions and disjunctions of contrast and orientation. On the other hand, the specific temporally serial model previously successfully used by Bergen and Julesz (1983a, 1983b) failed to predict human visual search accuracy for the conjunction displays (see Table 4B).

Our results are in disagreement with the results of Bergen and Julesz (1983a, 1983b) where the serial model predicted visual search accuracy for a T among Ls. Another disadvantage is that the serial model makes the same set-size prediction at all levels of target/distractor discriminability, unless one assumes that the number of items processed per second somehow depends on target/ distractor discriminability.

Figures $11 \mathrm{~A}-11 \mathrm{C}$ show that the SDT model can be used to successfully make predictions across display types. The ability to simultaneously predict performance for the conjunction and the disjunction displays shows that the difference in performance across displays can be accounted for by the model without assuming any qualitative or quantitative changes in the processing. Comparison between max-linear $d^{\prime}$ predictions for the modified Aiken and Palmer (1992) conjunction and disjunction displays and those for the standard conjunction and disjunction displays (see Table 3 ) show that $d^{\prime}$ in the former is larger by a factor of 1.25 for both displays. This find- 

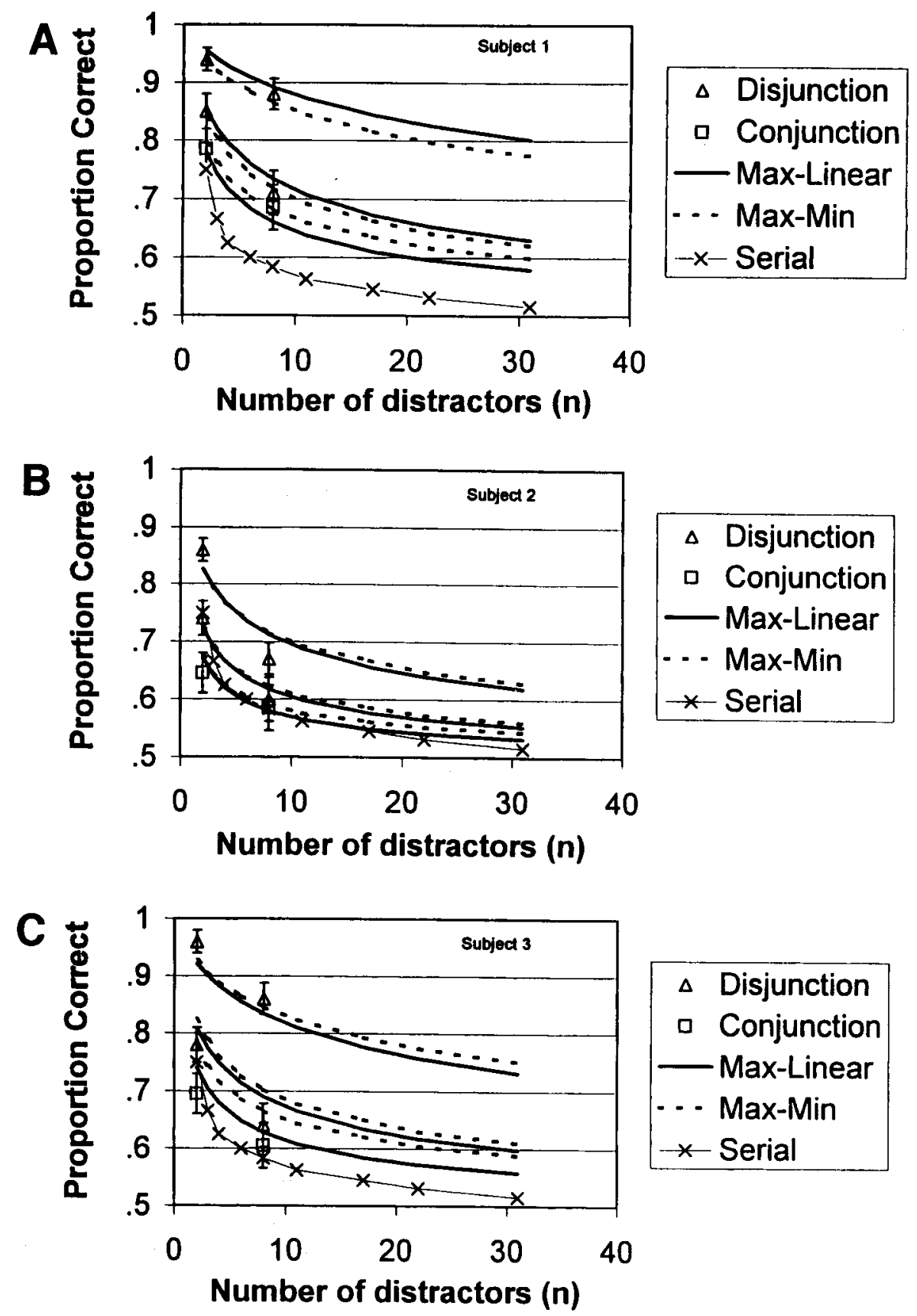

Figure 11. Proportion correct as a function of number of distractors in the display for three display conditions: conjunction, disjunction high target/distractor discriminability, and disjunction low target/distractor discriminability. There were two number-of-distractor conditions: $n=2$ and $n=8$. Solid lines correspond to the max-linear model used to fit the display conditions simultaneously. Dashed lines correspond to the max-min model used to fit all the display conditions simultaneously. Different figures $(A, B, C)$ correspond to the 3 different observers.

ing is consistent with the fact that the Aiken and Palmer displays included additional distractors (see Figure 10) that had higher target/distractor discriminability than did the original distractors in the standard conjunction and disjunction displays (see Figure 8). As a result, the group of distractors in the Aiken and Palmer displays degraded performance with respect to a feature display but less than did those in the standard conjunction and disjunction displays.

The ability to predict performance across the two disjunction conditions shows that the model can predict changes in set size with different levels of target/distractor 
Table 3

Predictions of the Max-Linear Model for the Standard Conjunction and Disjunction Displays and for the Modified Conjunction and Disjunction Displays Used by Aiken and Palmer (1992), Which Included an Additional Third Distractor Type

\begin{tabular}{ccccc}
\hline Feature & Conjunction & Modified Conjunction & Disjunction & Modified Disjunction \\
\hline$d_{\mathrm{o}}^{\prime}$ & $d_{0}^{\prime} / \sqrt{2}$ & $1.25 d_{0}^{\prime} / \sqrt{2}$ & $2 d_{0}^{\prime} / \sqrt{2}$ & $1.252 d_{0}^{\prime} / \sqrt{2}$ \\
\hline
\end{tabular}

discriminability $\left(d_{\mathrm{o}}^{\prime}\right)$ without assuming quantitative or qualitative changes in processing at the different levels of target/distractor discriminability. In the present treatment, we made the assumption that the internal discriminability between target and distractor was linear with the physical difference (contrast and orientation) between the target and distractor. The model fit might be improved if we were to explicitly measure the relationship between percentage of contrast difference or percentage of orientation difference and $d_{\mathrm{o}}^{\prime}$. Interestingly, the present results cannot be used to decide between the two decision strategies, max-linear or max-min. Further experiments need to be performed to decide which decision strategy best models human visual search accuracy.

\section{GENERAL DISCUSSION}

\section{Multidimensional Extension of the Signal Detection Theory Model}

We have presented an extension of an SDT based model that has been previously used to predict visual search accuracy in feature displays (Palmer, 1994a). Unlike most current theories of visual search, the SDT model provides a quantitative framework that can be used to rigorously test whether set-size effects are accounted for by the model. The model can be applied to predict the effects on visual search accuracy of target/ distractor similarity and distractor variability, number of distractors, and number of response alternatives, and it can be applied to a variety of multidimensional displays, including conjunction, disjunction, and triple conjunction displays (among others). Performance degradation is predicted with relatively few and well-established assumptions: internal noise, maximum response decision rule, independent processing of features, and for multidimensional displays, linear combination of responses or minimum response across feature dimensions.

Application of the model to data collected at the University of Washington showed how the model can be successfully used to predict performance in conjunctions and disjunctions of contrast and orientation. We have generated predictions for two different decision strategies for combining information across feature dimensions: the max-linear and max-min models. Throughout our theoretical treatment, we have assumed that the target/ distractor discriminabilities were matched across feature dimensions. This assumption guaranteed that the equal weighting in the linear combination was optimal. How- ever, the model needs to be generalized to the case in which the target/distractor discriminability is not approximately equal along the individual feature dimensions. We have also assumed that each feature dimension elicits a statistically independent response. Model predictions should be extended to the case in which there are possible correlations (nonindependent) between the feature responses.

\section{Feature Versus Conjunction}

There have been numerous studies finding larger setsize effects in search times for conjunction versus feature displays (Treisman \& Gelade, 1980; Wolfe et al., 1989). Duncan and Humphreys (1989) performed visual search time studies and showed that visual search efficiency increased with increasing target/distractor discriminability and decreased with increasing distractor variability. In their view, the feature/conjunction dichotomy was due to the lower target/distractor discriminability and the higher distractor variability in the conjunction displays. To determine whether target/distractor discriminability could explain all of the difference in performance, Treisman (1991) performed an experiment in which the discriminability between the target and the distractor was kept constant from the conjunction to the feature display. Starting with a conjunction display, this was achieved by eliminating the differences between target and distractors along one of the two feature dimensions and keeping the difference along the other dimen-

\section{Table 4}

Reduced Chi-Square Goodness of Fit $\left[\chi_{r}^{2}=\left(\chi^{2} / d f\right)\right]$ for Separate and Simultaneous Fits to the Different Display Conditions for the Two Signal Detection Theory Based Models and the Limited Capacity Serial Model of Bergen and Julesz (1983a, 1983b)

\begin{tabular}{lccc}
\hline & \multicolumn{3}{c}{ Chi-Square Fit } \\
\cline { 2 - 4 } Model & Subject I & Subject 2 & Subject 3 \\
Simultaneous Fit \\
Max-Linear & 1.10 & 2.23 & $6.1^{*}$ \\
Max-Min & 0.34 & 2.61 & $10^{*}$ \\
\multicolumn{4}{c}{ Separate Fit } \\
Max-Linear & \multicolumn{3}{c}{} \\
Conjunction & 0.358 & 6.48 & 2.46 \\
Disjunction 1 & 0.832 & 1.16 & 0.267 \\
$\quad$ Disjunction 2 & 0.516 & 0.97 & 0.56 \\
Serial & \multicolumn{3}{c}{} \\
$\quad$ Conjunction & $58.98^{*}$ & $41.93^{*}$ & $14.94^{*}$ \\
\hline
\end{tabular}

Note--Values with an asterisk $\left(^{*}\right)$ indicate that the model could be rejected at a .01 significance level. 
sion constant. Treisman still found larger set-size effects of conjunction displays versus feature displays where the target/discriminability $\left(d_{o}^{\prime}\right)$ along the feature dimensions in both displays were matched. Feature integration theory attributes the larger set-set size effect in conjunctions to the serial allocation of visual attention needed to bind information across feature dimensions.

Geisler and Chou (1995) have done experiments showing that the feature versus conjunction dichotomy could be explained, in part, by low-level factors. They conducted carefully controlled $2 \mathrm{AFC}$ search accuracy tasks and used the same target and background in a search time study. They showed that the rank order of the search times in feature and conjunction displays could be predicted from the size of an accuracy window. An accuracy window is defined as the accuracy $\left(P_{\mathrm{c}}\right)$ in a 2AFC as a function of target position eccentricity. They concluded that the feature/conjunction dichotomy can, in part, be accounted for by the low-level factors, such as stimulus information. Geisler and Chou did not manipulate set size, nor did they propose a mathematical model that generates visual search accuracy results as a function of number of distractors (set size). Their focus was on multiple fixation search tasks in which observers were allowed to move their eyes during the search. Their work also showed the effect of eccentricity on visual search accuracy.

The work presented in this paper specifically attempted to isolate set-size effects of an attentional nature from set-size effects from element eccentricity, element density, and eye movements. Therefore, we focused on an experimental paradigm that tried to neutralize other sources of set size.

Unlike previous treatments (e.g., Geisler \& Chou, 1995 ), our model explicitly attributes the feature/conjunction dichotomy to a specific neural computation. Our model predicts larger set-size effects for conjunction than for feature displays, even when the target/distractor discriminability along the individual feature dimensions are matched in the feature and conjunction displays (Treisman, 1991). In our model, the larger set-size effects for conjunctions are an emerging property of the system, given the existence of noise within the visual system, the independent processing of the features in the display, and an assumed decision rule for combining information across feature dimensions. Our results for disjunction (2-D feature) versus conjunction displays were well predicted by the SDT model and were not predicted by a serial attentional mechanism. Therefore, our findings with visual search accuracy are not consistent with a temporally serial mechanism that binds information across feature dimensions. These results agree with recent results (Eckstein, 1998) showing that the one-dimensional feature versus conjunction dichotomy is also predicted by the SDT-based model and not by a temporally serial mechanism.
The main assumption in the SDT model is that the two relevant search dimensions or features have independent noise. On the other hand, if the two features were processed by the same encoder and the observer had direct access to such an encoder, one might not expect the additional performance degradation in the conjunction display. This might be the case for conjunctions of stereo and motion. Nakayama and Silverman (1986) obtained experimental results for conjunction visual search efficiency no lower than that for the typical feature display and related the results to physiological evidence for cells in the visual cortex area MT that respond to motion and stereo disparity (Ballard, Hinton, \& Sejnowski 1983). The SDT model predicts that performance degradation from conjunctions can still be overcome by increasing the target/ distractor discriminability to higher levels. This observation seems to agree with results that found very shallow slopes (high search efficiency) for conjunction displays with high target/distractor discrıminability (Duncan \& Humphreys, 1989; McLeod, Driver, \& Crisp, 1988).

\section{Conjunction Versus Triple Conjunctions}

Wolfe et al. (1989) and also Quinlan and Humphreys (1987) found larger set-size effects in search time studies for conjunctions than for triple conjunctions where the target/distractor discriminabilities were kept constant across displays. Wolfe et al. interpreted the findings as supporting the idea of guided search where the parallel processes guide the serial-attention-mediated process (Wolfe, 1994; Wolfe et al., 1989). In this view, set-size effects are smaller in triple conjunctions, because in this case, the target differs from the distractors along two dimensions (vs. one dimension in standard conjunctions), providing more information by which the parallel process may guide attention. The proposed SDT model also predicts overall performance improvement and smaller set-size effects for visual search accuracy in the triple conjunction displays without resorting to a parallel mechanism's guiding of a serial mechanism.

It is also interesting to note that Wolfe et al. (1989) also performed experiments for a cacond kind of riple conjunctions in which the target differed from each of the distractors along a single feature dimension instead of two (e.g., a large, vertical, high-contrast ellipse target among large vertical, low-contrast ellipses, large, diagonal, highcontrast ellipses, and small vertical, high-contrast ellipses). His results showed that, for triple conjunctions in which targets and distractors differ along a single feature dimension, the set sizes are about the same size as those for conjunctions. Although we did not develop our model in detail for this display, it is interesting to observe that, for the max-linear model, if performance for such a display is transformed to $d_{0}^{\prime}$, using Equation 2, we obtain a $d^{\prime}$ that is smaller by a factor $\sqrt{3}$ than that for feature displays and about the same as the one obtained for conjunction displays (a factor of $\sqrt{2 / 3}$ smaller than the conjunc- 
Table 5

Comparison Between Predictions of the Signal Detection Theory (SDT)

Model for Set-Size Effects in Visual Search Accuracy Experiments

and Experimental Results for Search Accuracy and Search Time Studies

\begin{tabular}{llc}
\hline \multicolumn{1}{c}{ Predictions of the } & \multicolumn{2}{c}{ Experiments } \\
\cline { 2 - 3 } \multicolumn{1}{c}{ SDT Model for Set-Size Effects } & Search Accuracy & \multicolumn{1}{c}{ Search Times } \\
\hline $\begin{array}{l}\text { Target/distractor discriminability } \\
>\text { Set size effect }\end{array}$ & Palmer (1996) & Palmer (1996) \\
$\begin{array}{l}\text { Conjunction }>\text { feature } \\
\text { Geisler \& Chou (1995); }\end{array}$ & $\begin{array}{c}\text { Treisman \& Gelade (1980); } \\
\text { Duncan \& Humpheys (1989); } \\
\text { Geisler \& Chou (1995) }\end{array}$ \\
$\begin{array}{lll}\text { Conjunction }>\text { triple conjunction } \\
\text { Conjunction }>\text { disjunction (2-D feature) }\end{array}$ & $\begin{array}{l}\text { Bartroff \& Eckstein (1999) } \\
\text { Wesults in this paper }\end{array}$ & $\begin{array}{l}\text { Wolfe, Cave, \& Franzel (1989) } \\
\text { Treisman (1991) }\end{array}$ \\
\hline
\end{tabular}

Note-The SDT predictions in this table are for high performance levels. At low performance levels, these model predictions do not hold, because performance cannot degrade below chance level $(50 \%)$ for a twoalternative forced-choice task that leads to violations of the given predictions.

tion $d^{\prime}$ ). These results again seem to qualitatively agree with the findings of Wolfe et al.

\section{Search Accuracy Versus Search Times}

The presented SDT-based model makes predictions for visual search accuracy and makes no explicit predictions for reaction time search studies. In different parts of this paper, we compared the set-size effects predicted by the model in search accuracy studies with previous search time results in the literature. We found that, for high performance levels in the search accuracy studies, the rank order of set-size effects predicted by our model agreed closely with the results in search time studies. These comparisons included feature, conjunction, triple conjunction, and disjunction displays. Table 5 summarizes some of the set-size effects predicted by the presented SDT model for visual search accuracy with experimental results for visual search accuracy and visual search times.

The validity of the comparisons between search accuracy and search times is based on the assumption that larger set sizes in visual search accuracy studies lead to larger set sizes in visual search time studies. This assumption is supported by the experiments in Palmer (1995), where the target was a disk with a higher luminance than the distractors.

His results show larger set-size effects for the conditions in which the target/distractor difference in luminance is smaller. In a subsequent experiment with the same stimuli, the results show that the conditions with a smaller target/distractor luminance difference also lead to larger set-size effects in search time studies (with accuracy kept approximately constant; Palmer, 1996). This finding suggests that, within certain performance ranges, larger set-size effects in search accuracy studies lead to larger set-size effects in search time studies. Geisler and Chou (1995) presented experiments in which accuracy in a $2 \mathrm{AFC}$ and search times for the same stimuli as a function of eccentricity were measured. Their results also showed that lower performance in search accuracy studies led to larger response times in search time studies.

Even though these studies support the preservation of rank order of set sizes across displays, there is still a need for a model that can mathematically map accuracy measures to reaction times. Work extending the validity of the SDT theory from accuracy studies to reaction time studies has been recently reported (Palmer, 1994b, 1995, 1996; Palmer \& McLean, 1996).

\section{CONCLUSIONS}

We have extended and applied a model based on SDT to predict set-size effects on visual search accuracy for displays in which the target differs from the distractors along more than one dimension (multidimensional search displays). The model accounts for many findings in visual search without resorting to a temporally serial mechanism that binds information across feature dimensions.

\section{REFERENCES}

Aiken, D., \& Palmer, J. (1992). Pursuing an alternative theory of the difference between conjunctions and disjunctions in visual search. Unpublished master's thesis, University of Washington.

Ballard, D. H., Hinton, G. E., \& SeJnowski, T. J. (1983). Parallel visual computation. Nature, 306, 21-26.

BARLOW, H. B. (1957). Incremental thresholds at low intensities considered signals/noise discrimination. Journal of Physiology, 136, 469-488.

Bartroff, J. L., \& Eckstein, M. P. (1999). Improvement in search accuracy from feature to triple conjunctions is quantitatively predicted by signal detection theory. Investigative Ophthalmology \& Visual Science, 40 (Suppl.), 1835.

BENNETT, P. S., \& JAYE, P. D. (1995). Letter localization, not discrimination, is constrained by attention. Canadian Journal of Experimental Psychology, 49, 460-504.

Bergen, J. R., \& Julesz, B. (1983a). Parallel versus serial processing in rapid pattern discrimination. Nature, 303, 696-698.

BERGEN, J. R., \& Julesz, B. (1983b). Rapid discrimination of visual patterns. IEEE Transactions on Systems, Man, \& Cybernetics, 13, 857-863.

Burgess, A. E., \& Ghandeharian, H. (1984). Visual signal detection: II. Signal-location identification. Journal of the Optical Society of America A, 1, 906-910.

CAhILl, M. C., \& CARTER, R. C. (1976). Color size for searching displays of different density. Human Factors, 18, 273-280.

Carrasco M., Evert, D. L., Chang I., \& Katz, S. M. (1995). The eccentricity effect: Target eccentricity affects performance on conjunction searches. Perception \& Psychophysics, 57, 1241-1261.

Duncan J., \& HumphreYs, G. W. (1989). Visual search and stimulus similarity. Psychological Review, 96, 433-458. 
Eckstein, M. P. (1998). The lower efficiency for conjunctions is due to noise and not serial attentional processing. Psychological Science, 2 , 111-118.

Eckstein, M. P., Thomas, J. P., Palmer, J., \& Shimozaki, S. S. (1996). Further predictions of signal detection theory on visual search accuracy: Conjunction, disjunctions, and triple conjunctions [Abstract]. Investigative Ophthalmology \& Visual Science, 37 (Suppl.), 61.

Eckstein, M. P., Thomas, J. P., Shimozaki, S. S., \& Whiting, J. S. (1995). Signal detection theory explains many aspects of visual search efficiency. Investigative Ophthalmology \& Visual Science, 36 (Suppl.), 2994.

ECKSTEIN, M. P., \& Whiting, J. S. (1996). Visual signal detection in structured backgrounds: I. The effect of number of spatial locations and signal contrast. Journal of the Optical Society of America A, 9 , 1777-1787.

ESTES, W. K. (1972). Interactions of signal and background variables in visual processing. Perception \& Psychophysics, 12, 278-286.

FARMER, E. W., \& TAYLOR, R. M. (1980). Visual search through color displays: Effects of target-background similarity and background uniformity. Perception \& Psychophysics, 27, 267-272.

Geisler W. S., \& CHOU, K. L. (1995). Separation of low level and high level factors in complex tasks: Visual search. Psychology Review, 102, 356-378.

Green, D. M., \& Swets, J. A. (1966). Signal detection theory and psychophysics. New York: Krieger.

InDOW, T., \& KanAZAWA, K. (1960). Multidimensional mapping of colors varying in hue, chroma and value. Journal of Experimental $P_{s y-}$ chology, 59, 330-336.

MCLEOD, P., Driver, J., \& CRISP, J. (1988). Visual search for a conjunction of movement and form is parallel. Nature, 332, 154-155.

NAGY, A. L., \& SANCHEZ, R. R. (1990). Critical color differences determined with visual search task. Journal of the Optical Society of America, 7, 1209-1225.

Nakayama, K., \& Silverman, G. H. (1986). Serial and parallel processing of visual feature conjunctions. Nature, 320, 264-265.

Nolte, L. W., \& JAarsma, D. (1966). More on the detection of one of $\mathrm{M}$ orthogonal signals. Journal of the Acoustical Society of America, 41, 497.505.

Palmer, J. (1994a). Set-size effects in visual search: The effect of attention is independent of the stimulus for simple tasks. Vision Research, 34, 1703-1721.

Palmer, J. (1994b). Visual search latency: The influence of targetdistractor discriminability on the magnitude of set-size effects [Abstract]. Investigative Ophthalmology \& Visual Science, 35 (Suppl.), 2081 .

PALMER, J. (1995). Attention in visual search: Distinguishing four causes of set-size effects. Current Directions in Psychological Science, 4, 118-123.

PALMER, J. (1996). Attentional effects in visual search accuracy and search time. In R. Wright (Ed.), Visual attention (pp. 348-388). New York: Oxford University Press.

Palmer, J., AmEs, C. T., \& Lindsey, D. T. (1993). Measuring the effect of attention on simple visual search. Journal of Experimental Psychology: Human Perception \& Performance, 19, 108-130.

PALmer, J., \& MCLean, J. (1996). Visual search: Large set-size effects do not reject models based upon independent channels [Abstract]. Investigative Ophthalmology \& Visual Science, 37 (Suppl.), 62.

PASHLER, H. (1987). Target-distractor discriminability in visual search. Perception \& Psychophysics, 41, 285-292.

Pavel, M., Enconopouly, J., \& Landy, M. S. (1992). Psychophysics of rapid visual search. Paper presented at the meeting of the Association for Research in Vision and Ophthalmology, Sarasota, FL.

PelLI, D. G. (1985). Uncertainty explains many aspects of visual contrast detection and discrimination. Journal of the Optical Society of America A, 2, 1508-1532.

Quinlan, P. T., \& Humphreys, G. W. (1987). Visual search for targets defined by combinations of color, shape, and size: An examination of the task constraints on feature and conjunction searches. Perception \& Psychophysics, 41, 455-472.
SHAW, M. L. (1980). Identifying attentional and decision making components in information processing. In R. S. Nickerson (Ed.), Attention \& performance VIII (pp. 277-296). Hillsdale, NJ: Erlbaum.

SWENSSON, R. G., \& JUDY, P. F. (1981). Detection of noisy visual targets: Models for the effects of spatial uncertainty and signal-to-noise ratio. Perception \& Psychophysics, 29, 521-534.

SWETS, J. A. (Ed.) (1964). Signal detection and recognition by human observers: Contemporary readings. NewYork: Wiley.

TANNER, W. P., JR. (1961). Physiological implications of psychophysical data. (Annals of the New York Academy of Sciences, Vol. 89, p. 765). New York: New York Academy of Sciences.

TANner, W. P., JR., \& Swets, J. A. (1954). A decision-making theory of visual detection. Psychological Review, 61, 401-409.

TAYLOR, J. R. (1982). An introduction to error analysis. New York: Oxford University Press.

Tolmurst, D. J., Movshson, J. A., \& Dean, A. F. (1982). The statistical reliability of signals in single neurons in cat and monkey visual cortex. Vision Research, 23, 775-785.

TREISMAN, A. M. (1991). Search, similarity, and integration of features between and within dimensions. Journal of Experimental Psychology: Human Perception \& Performance, 17, 652-676.

Treisman, A. M., \& Gelade, G. (1980). A feature integration theory of attention. Cognitive Psychology, 12, 97-136.

Treisman, A. M., \& Gormican, S. (1988). Feature analysis in early vision: Evidence from dimensions. Journal of Experimental Psychology: Human Perception \& Performance, 17, 652-676.

Verghese, P., \& Nakayama, K. (1994). Stimulus discriminability in visual search. Vision Research, 34, 2453-2467.

Verghese, P., \& Stone, L. S. (1995). Combining speed information across space. Vision Research, 35, 2811-2823.

von Grünau, M., DubÉ, S., \& Galera, C. (1994). Local and global factors of similarity in visual search. Perception \& Psychophysics, 55, 575-592.

WiCKELGREN, W. A. (1968). Unidimensional strength theory and component analysis of noise in absolute and comparative judgments. Journal of Mathematical Psychology, 5, 102-122.

WOLFE, J. M. (1994). Guided Search 2.0: A revised model of visual search. Psychonomic Bulletin \& Review, 1, 202-238.

Wolfe, J. M., Cave, K. R., \& Franzel, S. L. (1989). Guided Search: An alternative to the feature integration model for visual search. Journal of Experimental Psychology: Human Perception \& Performance, 15, 419-433.

Zelinsky, G., SheinberG, D., \& Bulthoff, H. H. (1993). Eye movements during visual search [Abstract]. Investigative Ophthalmology \& Visual Science, 34 (Suppl.), 1236.

\section{NOTES}

1. The statement does not hold for low discriminability $\left(d_{0}^{\prime}=1.0\right.$ and 0.5 ), where performance levels cannot decrease below the $50 \%$ chance level.

2. However, for the minimum operation to be general, a common scale is needed to compare values across feature dimensions. In order to guarantee the generality and effectiveness of the max-min decision rule described, one has to take three steps. (1) We adjust the sign of the scale along the feature dimensions so that the target always elicits that larger response. (2) From each element's individual feature response, we subtract the mean response of the distractor type with the smallest mean feature response. (3) We divide the feature responses by the internal noise in that feature dimension. This step transforms the responses along different feature dimensions to a common scale, signal-to-noise ratio.

3 . The derived equation can also be applied to one-dimensional displays with distractor variability, such as those studied by others (Cahill \& Carter, 1976; Indow \& Kanazawa, 1960).

4. This can be achieved by performing individual feature search experiments (high-contrast vertical ellipse among low-contrast ellipses and a vertically oriented high-contrast ellipse among nonvertically oriented high-contrast ellipses) and adjusting the target/distractor physical difference along the relevant feature dimension in order to achieve ap- 
proximately the same performance across all feature conditions (e.g., orientation feature search, contrast feature search).

5. Note that, for the disjunction display, a max-min rule results in the same predictions as a max-max rule (an observer who, for each element, takes the maximum response among the two feature dimension responses and then chooses the display containing the maximum response among the maximum feature responses).

6 . The mean and variance for the two distractors and the target in Treisman's (1991) 2-D feature display can be written to be: Target: $\bar{x}_{1}, \bar{x}_{2}, \sigma_{0}^{2}$; Distractor 1: $\bar{x}_{1}+u_{\mathrm{o}}, \bar{x}_{2}+u_{\mathrm{o}}, \sigma_{0}^{2}$; Distractor $2: \bar{x}_{1}-u_{\mathrm{o}}, \bar{x}_{2}-$ $u_{0}, \sigma_{0}^{2}$. Given that the target does not have the larger response value, the max-linear and the max-min decision rules are not directly applicable to this task. However, one can apply a transformation to the responses by subtracting from all responses by the expected mean responses for the target, then taking the absolute value of the responses and finally the negative of the responses. After this transformation the mean and variance of the responses for target and distractors become Target: $-\left|\bar{x}_{1}-\bar{x}_{1}\right|=0,-\left|\bar{x}_{2}-\bar{x}_{2}\right|=0, \sigma_{0}^{2}$; Distractor 1: $-u_{0},-u_{0}, \sigma_{0}^{2}$; Distractor 2 : $-u_{0},-u_{0}, \sigma_{0}^{2}$. With the transformation, the expected responses for the two distractors become the same, and the Treisman (1991) 2-D feature display is equivalent to the 2-D feature disjunction display presented in this paper. The model predictions for both 2-D feature cases are identical.

7. For the max-linear model, matching performance between the feature condition and the multidimensional display would involve increasing the target/distractor discriminability $\left(d_{0}^{\prime}\right)$ along each feature dimension by a factor of $\sqrt{2}$ for the conjunction display and $\sqrt{3} / 2$ for the triple conjunction display and reducing it by a factor of $\sqrt{2} / 2$ for the disjunction display.

\section{APPENDIX A \\ Feature Displays}

The probability of correct identification of the target-present display $\left(P_{\mathrm{c}}\right)$ is the probability that the maximum response to the target-present display is larger than the maximum response to the target-absent display. Assuming that each element elicits a statistically independent internal response, $P_{\mathrm{c}}$ can be expressed as the product of the probability that the maximum response to the target-present display will take a value $x$ and the probability that the maximum response to the $M-1$ target-absent displays will take a value less than $x$ :

$$
P_{\mathrm{c}}=P\left[\max \left(T, D_{t 1}, D_{t 2}, \ldots, D_{t n-1}\right)=x\right] P\left[\max \left(D_{d 1}, D_{d 2}, \ldots, D_{d n}\right)<x\right], \text { for all } x .
$$

The probability that the maximum response to elements in the target-present display will take a value $x$ can be expressed as the sum of $t(x) D^{n-1}(x)$ (the first term in Equation A2; the probability that the target will take a value $x$ and that the $n-1$ distractors will take values less than $x),(n-1) d(\mathrm{x})$ (the probability that any one of the $n-1$ distractors [ $n-1$ permutations] will take a value $x$ ), and $T(x) D^{n-2}(x)$ (second term in Equation A2; the probability that the target and the remaining $n-2$ distractors will take values less than $x$ ):

$$
P\left[\max \left(T, D_{t 1}, D_{t 2}, \ldots, D_{t n-1}\right)=x\right]=t(x) D^{n-1}(x)+(n-1) \cdot d(x) D^{n-2}(x) T(x) .
$$

The probability that the maximum response in the target-absent display will take a value less than $x$ can be guaranteed if all the distractors take a value less than $x$ :

$$
P\left[\max \left(D_{d 1}, D_{d 2}, \ldots, D_{d n}\right)<x\right]=\prod_{1}^{n} D(x)=D^{n}(x) .
$$

Replacing expressions $\mathrm{A} 2$ and $\mathrm{A} 3$ into Equation $\mathrm{A} 1$ and integrating over all possible values of $x$, we obtain

$$
P_{\mathrm{c}} \int_{-\infty}^{+\infty}\left[t(x) D^{n-1}(x)+(n-1) d(x) D^{n-2}(x) T(x)\right] D^{n}(x) d x .
$$

The equation can be generalized from a $2 \mathrm{AFC}$ to any $M$-AFC by noting that, in an $M$-AFC, there are $M-1$ target-absent displays containing $n$ distractors that cannot exceed the maximum response to the target-present display:

$$
P_{\mathrm{c}}(M, n)=\int_{-\infty}^{+\infty}\left[t(x) D^{n-1}(x)+(n-1) d(x) D^{n-2}(x) T(x)\right]\left[D^{n}(x)\right]^{M-1} d x .
$$

With the assumption of equal Gaussian internal noise for the target and distractors, the target and distractor density functions are described with equal Gaussian variance distributions, and Equation A5 can be written as

$$
P_{\mathrm{c}}\left(M, n, d_{\mathrm{o}}^{\prime}\right)=\int_{-\infty}^{+\infty}\left[g\left(x-d_{\mathrm{o}}^{\prime}\right) G^{n-1}(x)+(n-1) g(x) G^{n-2}(x) G\left(x-d_{\mathrm{o}}^{\prime}\right)\right]\left[G^{n}(x)\right]^{M-1} d x,
$$

where $M$ is the number of alternatives, $n$ the number of distractors per alternative, $d_{0}^{\prime}$ is the distance between the target and the distractor distribution in standard deviation units, $g(x)$ is the probability of the Gaussian distribution's taking a value $x$,

$$
g(x)=\sqrt{1 / 2 \pi} \exp \left(\frac{-x^{2}}{2}\right)
$$

and $G(x)$ is the cumulative probability of the Gaussian distribution taking a value less than

$$
G(x)=\int_{-\infty}^{x} \sqrt{1 / 2 \pi} \exp \left(\frac{-y^{2}}{2}\right) d y .
$$




\section{APPENDIX A (Continued)}

When there are no distractors in the target-present display $(n=1)$, Equation A4 reduces to the familiar 2AFC expression, with one display containing one nontarget and the other the target (Green \& Swets, 1966):

$$
P_{c}(M)=\int_{-\infty}^{+\infty} t(x)[D(x)]^{M-1} d x
$$

A mathematical expression by which to calculate $P_{\mathrm{c}}$ in the feature condition was first derived by Shaw (1980) and is mathematically equivalent to Equation A1.

\section{APPENDIX B}

\section{Combining Information Across Feature Dimensions}

\section{Max-Linear Decision Rule}

In order to calculate the effective target/distractor discriminability along the new decision variable, we must find the expected value of the mean response and standard deviation to the target and distractors after the linear combination of responses across feature dimensions. The mean response to an element along the new decision variable will be the linear combination of the mean responses of that element along the individual feature dimensions:

$$
\bar{Z}_{i}=\sum_{j=1}^{f} w_{j} \cdot \bar{x}_{i j} .
$$

The variance of an element's response along the new decision variable, $\sigma_{z}^{2}$, is given by (Taylor, 1982)

$$
\sigma_{z i}^{2}=\sum_{j=1}^{\prime}\left[\frac{\partial Z}{\partial X_{i j}}\right]^{2} \sigma_{x_{i j}}^{2},
$$

where

$$
\left[\frac{\partial Z}{\partial X_{i j}}\right]
$$

is the partial derivatives of $Z$ with respect to $x_{i j}$ and $\sigma_{x_{i j}}^{2}$ is the variance of the responses along the $j$ th dimension. For the case of a linear combination of responses across feature dimensions, the variance of the new decision variable, $\sigma_{z}^{2}$, reduces to

$$
\sigma_{z i}^{2}=\sum_{j=1}^{f} w_{j}^{2} \cdot \sigma_{x_{i j}}^{2},
$$

The internal discriminability between a single target and the $i$ th distractor along the new decision variable can then be described by the distance between the means of the target distribution $\left(\bar{Z}_{t}\right)$ and distractor distribution $\left(\bar{Z}_{d i}\right)$ divided by the standard deviation of the distributions:

$$
d_{i=}^{\prime}=\frac{\bar{Z}_{t}-\bar{Z}_{d i}}{\sqrt{\sigma_{z}^{2}}} .
$$

For the case in which the standard deviation for internal noise is the same along the different feature dimensions and for the different elements, and replacing B1 and B3 into B4, the effective target/distractor discriminability reduces to

$$
d_{i z}^{\prime}=\frac{\sum_{j=1}^{f} w_{j} d_{i j}^{\prime}}{\sum_{j=1}^{f} w_{j}^{2}},
$$

where

$$
d_{i j}^{\prime}=\frac{\bar{x}_{t_{j}}-\bar{x}_{d_{i j}}}{\sigma_{j}}
$$

is the discriminability between the target and the $i$ th distractor along the $j$ th feature dimension.

For the special case in which the target/distractor discriminabilities along all $r$ feature dimensions are approximately equal and the observer uses equal weighting ( $w_{j}=1$ for all $j$ s) for all $f$ feature dimensions, Equation $\mathrm{B} 5$ reduces to 
APPENDIX B (Continued)

$$
d_{r, f}^{\prime}=\frac{r d_{\mathrm{o}}^{\prime}}{\sqrt{f}}
$$

where $d_{0}^{\prime}$ is the target/distractor discriminability along the individual feature dimensions, $r$ is the number of features along which the target differs from the distractors, and $f$ is the number of features information is combined across.

\section{Max-Min Decision Rule}

A second possible way in which the observer can combine information across feature dimensions for each element is by choosing the minimum response across feature dimensions. Given that each element consists of many feature dimensions, the distribution of the minimum is given by the probability that the $i$ th element will take a value $x$ along the $j$ th feature dimension $\left[e_{i j}(x)\right]$ times the probability that all other responses along the remaining feature dimensions take a value larger than $x$,

$$
\begin{gathered}
\prod_{k=1}^{f}\left[E_{i k}(>x)\right]^{\left(1-\delta_{j k}\right)}: \\
e_{i, \min }(x)=\operatorname{Min}\left\{x_{i j}, j=1, \ldots, f\right\}=\sum_{j=1}^{f} e_{i j}(x) \cdot \prod_{k=1}^{f}\left[E_{i k}(>x)\right]^{\left(1-\delta_{j k}\right)} .
\end{gathered}
$$

Specializing the equation for the target and distractors, we obtain

$$
t_{\min }(x)=\operatorname{Min}\left\{x_{i}, i=1, \ldots, f\right\}=\sum_{j=1}^{f} t_{j}(x) \cdot \prod_{k=1}^{f}\left[T_{k}(>x)\right]^{\left(\mathrm{l}-\delta_{j k}\right)}
$$

and

$$
d_{\min }(x)=\operatorname{Min}\left\{x_{i}, i=1, \ldots, f\right\}=\sum_{j=1}^{f} d_{j}(x) \cdot \prod_{k=1}^{f}\left[D_{k}(>x)\right]^{\left(1-\delta_{j k}\right)} .
$$

Assuming that the target differs from the distractors along $r$ of the $f$ distractors $(f \geq r)$ and that the target/ distractor discriminability along all those $r$ feature dimensions is the same, Equations B7 and B8 become

$$
t_{\min }(x)=f \cdot t(x)[T(>x)]^{(f-1)}
$$

and

$$
d_{\min }(x)=(f-r) t(x) T(>x)^{f-r-1} D(>x)^{r}+r d(x) D(>x)^{r-1} T(>x)^{f-r} .
$$

With the assumption that the internal responses are Gaussian distributed

and

$$
t(x)=g\left(x-d_{0}^{\prime}\right), d(x)=g(x), T(x)=\int_{x}^{+\infty} t(y) d y,
$$

$$
D(x)=\int_{x}^{+\infty} d(y) d y
$$

\section{APPENDIX C \\ Multiple Distractor Types (Distractor Variability)}

For the case of multiple distractor types with different associated target/distractor discriminability along the new decision variable, the probability of correct identification of the target-present display is still the probability that the maximum response to the target-present display will be larger than the maximum response to the target-absent display (Equation A1). The only difference is that now there are a total of $k$ different distractors types, instead of one type of distractors. Each $i$ th distractor type has $n_{i}$ elements. The probability that the maximum response to elements in the target-present display will take a value of $x$ can be expressed as the sum of

$$
t(x) \prod_{i=1}^{k} D_{i}^{n_{i}}(x)
$$

(the first term in $\mathrm{C} 1$ ), the probability that the target will take a value of $x$ and that the $n_{i}$ distractors of all $k$ types will take values less than $x$, and 


\section{APPENDIX C (Continued)}

$$
n_{i} d_{i}(x) T(x) D_{i}^{n_{i}-1}(x) \prod_{j=1}^{k}\left[D_{i}^{n_{i}-1}(x)\right]^{\left(1-\delta_{j}\right)}
$$

(the second term in $\mathrm{Cl}$; also, $\delta_{i j}$ is Kronecker delta, which is 1 for $i=j$ and 0 otherwise), the probability that any one of the $n_{i}$ distractors [ $n_{i}$ permutations] of all $k$ distractor types will take a value of $x$ $\left[n_{i} d_{i}(x)\right]$, that the target and all the remaining $n_{i}-1$ distractors of the $i$ th type will take a value less than $x\left[T(x) D_{i}^{n_{i}-1}(x)\right]$, and that all the $n_{i}$ distractors of the $k-1$ remaining types will take a value less than $x$

$$
\begin{gathered}
{\left[\prod_{j=1}^{k}\left[D_{i}^{n_{i}}(x)\right]^{\left(1-\delta_{i, 1}\right)}\right]:} \\
P\left[\max \left(T, D_{t 1}, D_{t 2}, \ldots, D_{t n}\right)=x\right]=t(x) \prod_{i=1}^{k} D_{i}^{n_{i}}(x)+\sum_{i=1}^{k}\left[n_{i} \cdot d_{i}(x) \cdot T(x) \cdot D_{i}^{n_{i}-1}(x) \prod_{j=1}^{k} \cdot\left[D_{i}^{n_{i}}(x)\right]^{\left(1-\delta_{i j}\right)}\right] .
\end{gathered}
$$

The probability that the maximum response in the target-absent displays will take a value less than $x$ can be guaranteed if all the distractors take a value less than $x$ :

$$
P\left[\max \left(D_{d 1}, D_{d 2}, \ldots, D_{d n}+1\right)<x\right]=\prod_{i=1}^{k+1} D_{i}^{n_{i}}(x)
$$

where $D_{i}^{n_{i}}(x)$ is the cumulative probability that all the $n_{i}$ distractors of $i$ th type take a value less than $x$. Note that the multiplication index runs to $k+1$, because of an additional distractor in the target-absent display that was added so that the target-present and the target-absent displays would contain the same number of elements.

Replacing expressions $\mathrm{C} 1$ and $\mathrm{C} 2$ into Equation $\mathrm{Al}$ and integrating over all possible values of $x$, we obtain

$$
\begin{aligned}
P_{\mathrm{c}}(N, M, k)= & \int_{-\infty}^{+\infty}\left[t(x) \prod_{i=1}^{k} D_{i}^{n_{i}}(x)+\sum_{i=1}^{k}\left[n_{i} \cdot d_{i}(x) \cdot T(x) \cdot D_{i}^{n_{i}-1}(x) \prod_{j=1}^{k} \cdot\left[D_{i}^{n_{j}}(x)\right]^{\left(1-\delta_{j}\right)}\right]\right] \\
& \cdot\left[\prod_{i=1}^{k+1} D_{i}^{n_{i}}(x)\right]^{M-1} d x .
\end{aligned}
$$

$P_{\mathrm{c}}(N, M, k)$ is performance for $M$ alternatives and $k$ distractors types, where the number of distractors $n_{i}$ of each of the $i$ th type is given by $N=\left\{n_{1}, n_{2}, n_{3}, \ldots n_{k}\right\}$ and $\delta_{i j}$ is Kronecker delta, as defined above.

If the observers' internal responses to the different elements in the display are assumed to be Gaussian and only different in their mean internal response, the probability distributions of the responses for the different elements can be parameterized in terms of the distances in standard deviation units between the target response distribution and each of the distractor response distributions $\left(d_{i}^{\prime}\right)$. Equation $\mathrm{C} 3$ then reduces to

$$
\begin{aligned}
P\left(N, M, k, D^{\prime}\right)= & \int_{-\infty}^{+\infty}\left[g(x) \prod_{i=1}^{k} G^{n_{i}}\left(x+d_{i}^{\prime}\right)\right. \\
& \left.+\sum_{i=1}^{k}\left\lfloor n_{i} \cdot g\left(x+d_{i}^{\prime}\right) \cdot G(x) \cdot G^{n_{i}-1}\left(x+d_{i}^{\prime}\right) \prod_{j=1}^{k}\left[G^{n_{j}}\left(x+d_{j}^{\prime}\right)\right]^{\left(1-\delta_{i j}\right)}\right]\right] \\
& \cdot\left[\prod_{i=1}^{k+1} G^{n_{i}}\left(x+d_{i}^{\prime}\right)\right]^{M-1} d x,
\end{aligned}
$$

where $P\left(N, M, k, D^{\prime}\right)$ is the $P_{\mathrm{c}}$ for performance as a function of $k$ (the total number of distractor types), the number of distractors $n_{i}$ of the $i$ th distractor type as specified by $N=\left\{n_{1}, n_{2}, n_{3}, \ldots n_{k}\right\}$, the number of response alternatives $M$, and $d_{i}^{\prime}$ (the discriminability between the target and the $i$ th distractor type as specified by the set $\left.D^{\prime}=\left\{d_{1}^{\prime}, d_{2}^{\prime}, d_{3}^{\prime}, \ldots d_{k}^{\prime}\right\}\right)$. The set size is the total number of distractors plus the target and is given by

$$
n=\sum_{i=1}^{k} n_{i}+1,
$$

where the summation is over $k$ the total number of distractor types. Also, $g(x)$ and $G(x)$ are defined as in Equations $\mathrm{A} 7$ and $\mathrm{A} 8$. 


\section{APPENDIX D \\ Derivation of Model Predictions for the Aiken and Palmer (1992) Experiment}

\section{Max-Linear Decision Rule}

The first step needed to calculate $P_{\mathrm{c}}$ is to obtain the effective target/distractor discriminability along the new decision variable after the linear combination of information across feature dimensions. Assuming that the observer equally weights the information across feature dimensions and taking into account that the target/distractor discriminability was matched along the orientation and contrast dimensions, we can use Equation B5 to obtain the target/distractor discriminability along the new decision variable $\left(d_{i z}^{\prime}\right)$.

Modified conjunction task. For two of the distractors in the modified conjunction, $d_{1 z}^{\prime}=d_{2 z}^{\prime}$ and is given by

$$
d_{1 z}^{\prime}=d_{2 z}^{\prime}=\frac{\sum_{j=1}^{f} d_{i j}^{\prime}}{\sqrt{f}}=\frac{\left[d_{\mathrm{o}}^{\prime}+0\right]}{\sqrt{2}}=1_{\sqrt{2}} d_{\mathrm{o}}^{\prime} .
$$

And for the third distractor,

$$
d_{3 z}^{\prime}=\frac{\sum_{j=1}^{f} d_{1 j}^{\prime}}{\sqrt{f}}=\frac{\left[d_{\mathrm{o}}^{\prime}+d_{\mathrm{o}}^{\prime}\right]}{\sqrt{2}}=2_{\sqrt{2}} d_{\mathrm{o}}^{\prime},
$$

where $d_{0}^{\prime}$ is the target/distractor discriminability between the target and distractors along the individual feature dimensions (contrast and orientation).

Modified disjunction task. Similarly, we can obtain the effective target/distractor discriminability for the modified disjunction display:

$$
d_{1 z}^{\prime}=d_{2 z}^{\prime}=\frac{\sum_{j=1}^{f} d_{1 j}^{\prime}}{\sqrt{f}}=\frac{\left[2 d_{\mathrm{o}}^{\prime}+d_{\mathrm{o}}^{\prime}\right]}{\sqrt{2}}=3_{\sqrt{2}} d_{\mathrm{o}}^{\prime}
$$

and

$$
d_{3 z}^{\prime}=\frac{\sum_{j=1}^{f} d_{1 j}^{\prime}}{\sqrt{f}}=\frac{\left[d_{\mathrm{o}}^{\prime}+d_{\mathrm{o}}^{\prime}\right]}{\sqrt{2}}=2_{{ }_{2}} d_{\mathrm{o}}^{\prime} .
$$

Calculation of Proportion Correct. Since the modified conjunctions and disjunction displays contain multiple distractors with different associated target/distractor discriminability along the new decision variable, calculating $P_{\mathrm{c}}$ requires equation $\mathrm{C} 4$ (for multiple distractors), rather than Equation A6. The expressions for $d_{1 z}^{\prime}, d_{2 z}^{\prime}$, and $d_{3 z}^{\prime}$ for the conjunction and disjunction displays (Equations D1-D4) might be used with equation $\mathrm{C} 4$ in order to predict $P_{\mathrm{c}}$. However, an additional property of the Aiken and Palmer (1992) studies was that, on each trial, the selection of a particular distractor was probabilistic. In this way, the distractor configuration varied from trial to trial. In this way, specializing Equation $\mathrm{C} 4$ for any particular distractor configuration will not correctly describe $P_{\mathrm{c}}$ for the Aiken and Palmer experiments. The appropriate expected value of $P_{\mathrm{c}}$ for these experiments, in which the distractor configuration was randomly determined from trial to trial, is calculated by calculating the $P_{\mathrm{c}}$ for each possible display configuration and multiplying it by the probability of occurrence of that particular display:

$$
E\left(P_{\mathrm{c}}\right)=p_{1} \cdot P_{\mathrm{c} 1}+p_{2} \cdot P_{\mathrm{c} 2}+p_{3} \cdot P_{\mathrm{c} 3}+\ldots+p_{k} \cdot P_{\mathrm{c}_{k}}=\sum_{i=1}^{k} p_{i} \cdot P_{\mathrm{c}_{i}},
$$

where $E\left(P_{\mathrm{c}}\right)$ is the expectation value of $P_{\mathrm{c}}$ for the experiment, $P_{\mathrm{c}_{i}}$ is the $P_{\mathrm{c}}$ prediction for the $i$ th display configuration, and $p_{i}$ is the probability of occurrence of the $i$ th display.

Noting that the probability of occurrence of a given distractor configuration with $i$ Distractors 1 and $n-1-i$ Distractors 2 is given by (Taylor, 1982)

$$
\left(\begin{array}{c}
n-1 \\
i
\end{array}\right) p_{d 1}^{i}, p_{d 2}^{n-1-i}
$$

a general equation describing the expectation value of $P_{\mathrm{c}}$ as a function of number of distractors and $d_{\mathrm{o}}^{\prime}$ can be derived:

$$
E\left(P_{\mathrm{c}}\left(n, 2, d_{o}^{\prime}\right)=\sum_{j=0}^{n} \sum_{i=0}^{n-1}[I(n, i)+J(n, i)+K(n, i)] \cdot[L(n, j)],\right.
$$




\section{APPENDIX D (Continued)}

where the summations are over the number of distractors of Type 1 in the target-present and the targetabsent displays.

Also, the expressions $I(n, i), J(n, i), K(n, i), L(n, j)$ are given as follows.

$$
I(n, i)=\int_{-\infty}^{+\infty}\left(\begin{array}{c}
n-1 \\
i
\end{array}\right) g(z)\left(\frac{2}{3}\right)^{i} G^{i}\left(z+d_{z 1}^{\prime}\right)\left(\frac{1}{3}\right)^{n-1-i} G^{n-1-i}\left(z+d_{z 2}^{\prime}\right) d z
$$

is the probability that the target will obtain a value of $z$ and the probability that all the $i$ Distractors 1 and the $n-1-i$ distractors will be less than $z$;

$$
J(n, i)=\int_{-\infty}^{+\infty}\left(\begin{array}{c}
n-1 \\
i
\end{array}\right) \cdot i\left(\frac{2}{3}\right) g\left(z+d_{z 1}^{\prime}\right)\left(\frac{2}{3}\right)^{i-1} G^{i-1}\left(z+d_{z 1}^{\prime}\right)\left(\frac{1}{3}\right)^{n-i-1} G^{n-1-i}\left(z+d_{z 2}^{\prime}\right) G(z) d z
$$

is the probability that any one Distractor 1 will take a value of $z$ and that the $i-1$ Distractors 1 , the $n-$ $1-i$ Distractors 2, and the target will be less than $z$;

$$
K(n, i)=\int_{-\infty}^{+\infty}\left(\begin{array}{c}
n-1 \\
i
\end{array}\right) \cdot(n-1-i) \cdot\left(\frac{1}{3}\right) g\left(z+d_{z 2}^{\prime}\right)\left(\frac{1}{3}\right)^{n-i-2} G^{n-i-2}\left(z+d_{z 2}^{\prime}\right)\left(\frac{2}{3}\right)^{i} G^{i}\left(z+d_{z 1}^{\prime}\right) G(z) d z
$$

is the probability that any one Distractor 2 will take a value of $z$ and that the $n-i-2$ Distractors 2 , the $i$ Distractors 1 , and the target will be less than $z$; and

$$
L(n, j)=\int_{-\infty}^{+\infty}\left(\begin{array}{l}
n \\
j
\end{array}\right)\left(\frac{2}{3}\right)^{j} G^{j}\left(z+d_{z 1}^{\prime}\right)\left(\frac{1}{3}\right)^{n-j} G^{n-j}\left(z+d_{z 2}^{\prime}\right) d z
$$

is the probability that all the $j$ Distractors 1 and the $n-j$ Distractors 2 in the target-absent display will take a value of less than $z$ (the maximum of the target-present display).

The validity of Equation D10 was verified with Monte Carlo simulations performed in the same fashion as those described previously.

\section{Max-Min Decision Rule}

Performance for the max-min observer for the Aiken and Palmer (1992) experiment can be found by first computing the probability of the minimum responses in the target present and target-absent displays. Replacing the probability distributions for the responses of each element type along both feature dimensions into Equations B8 and B9 we obtain the following.

Modified conjunction. For the conjunction task, the target minimum response distribution is given by

$$
\begin{aligned}
& t_{\text {min }}(x)=g\left(x-d_{\mathrm{o}}^{\prime}\right) \cdot G\left(>x-d_{\mathrm{o}}^{\prime}\right)+g\left(x-d_{\mathrm{o}}^{\prime}\right) \cdot G\left(>x-d_{\mathrm{o}}^{\prime}\right), \\
& d_{1_{\min }}(x)=d_{2_{\min }}(x)=g\left(x-d_{\mathrm{o}}^{\prime}\right) \cdot G(>x)+g(x) \cdot G\left(x-d_{\mathrm{o}}^{\prime}\right), \\
& d_{3_{\min }}(x)=g(x) \cdot G(>x)+g(x) \cdot G(>x) .
\end{aligned}
$$

Modified disjunction. For the disjunction task, the target minimum response distribution is given by

$$
\begin{aligned}
t_{\text {min }}(x) & =g\left(x-d_{\mathrm{o}}^{\prime}\right) \cdot G\left(>x-d_{\mathrm{o}}^{\prime}\right)+g\left(x-d_{\mathrm{o}}^{\prime}\right) \cdot G\left(>x-d_{\mathrm{o}}^{\prime}\right), \\
d_{1_{\text {min }}}(x) & =d_{2_{\text {min }}}(x)=g\left(x+d_{\mathrm{o}}^{\prime}\right) \cdot G(>x)+g(x) \cdot G\left(>x+d_{\mathrm{o}}^{\prime}\right), \\
d_{3_{\text {min }}}(x) & =g(x) \cdot G(>x)+g(x) \cdot G(>x),
\end{aligned}
$$

and

$$
D_{i \min }(x)=\int_{-\infty}^{x} d_{i \min }(y) d y \text { and } T_{\min }(x)=\int_{-\infty}^{x} t_{\min }(y) d y,
$$

where $t_{\min }(x)$ is the probability function of the target's minimum response between the two feature dimensions, $d_{i \min }(x)$ is the probability function of the distractors ' minimum response between the two feature dimensions, $D_{i \min }(x)$ is the cumulative probability that the distractors' minimum response between the two feature dimensions will take a value less than $x$, and $T_{\min }(x)$ is the cumulative probability that the target's minimum response between the two feature dimensions will take a value of less than $x$.

Plugging in the expressions for $t_{\min }$ and $d_{i \min }, T_{\min }$ and $D_{i \min }$ into equations similar to D6-D10, one can obtain an expression for $P_{\mathrm{c}}$ as a function of number of distractors $(n)$, number of response alternatives $(M)$, and target/distractor discriminability $\left(d_{0}^{\prime}\right)$ for the max-min observer. The equations were verified with a Monte Carlo simulation in the same fashion as that described previously. 